\title{
Towards the first targeted therapy for triple-negative breast cancer: repositioning of clofazimine as a chemotherapy- compatible selective Wnt pathway inhibitor
}

Kamal Ahmed $^{1, \#}$, Alexey Koval ${ }^{1,2, \#}$, Jiabin $\mathrm{Xu}^{1,2}$, Alexandre Bodmer ${ }^{3}$ and Vladimir L. Katanaev ${ }^{2,4, *}$

${ }^{1}$ Department of Pharmacology and Toxicology, Faculty of Biology and Medicine, University of Lausanne, Lausanne, Switzerland;

${ }^{2}$ Department of Cell Physiology and Metabolism, Translational Research Centre in Oncohaematology, Faculty of Medicine, University of Geneva, Geneva, Switzerland;

${ }^{3}$ Department of Oncology, Geneva University Hospital, Geneva, Switzerland;

${ }^{4}$ School of Biomedicine, Far Eastern Federal University, Vladivostok, Russia.

\#these authors contributed equally to this work.

*correspondence to: vladimir.katanaev@unige.ch 


\section{Abstract}

Wnt signaling is overactivated in triple-negative breast cancer (TNBC) and several other cancers, and its suppression emerges as an effective anticancer treatment. However, no drugs targeting the Wnt pathway exist on the market nor in advanced clinical trials. Here we provide a comprehensive body of preclinical evidence that an anti-leprotic drug clofazimine is effective against TNBC. Clofazimine specifically inhibits canonical Wnt signaling in a panel of TNBC cells in vitro. In several mouse xenograft models of TNBC, clofazimine efficiently suppresses tumor growth, correlating with in vivo inhibition of the Wnt pathway in the tumors. Clofazimine is well compatible with doxorubicin, exerting additive effects on tumor growth suppression, producing no adverse effects. Its excellent and wellcharacterized pharmacokinetics profile, lack of serious adverse effects at moderate (yet therapeutically effective) doses, its combinability with cytotoxic therapeutics, and the novel mechanistic mode of action make clofazimine a prime candidate for the repositioning clinical trials. Our work may bring forward the anti-Wnt targeted therapy, desperately needed for thousands of patients currently lacking targeted treatments.

Keywords: triple-negative breast cancer; clofazimine; Wnt signaling; targeted therapy; drug combination 


\section{Introduction}

Wnt signaling is a highly evolutionary conserved developmental signal transduction pathway, which controls multiple events during embryogenesis regulating cell proliferation, differentiation, motility, migration and polarization [1]. No wonder that the Wnt pathway, as it is the case for many other developmental pathways, is frequently hijacked by cancer cells that employ most of the abovementioned features for promotion of tumor growth, metastasis and drug resistance. Targeting of the Wnt pathway is therefore an attractive option for development of new anticancer drugs [2, 3]. However, many of the anti-Wnt compounds failed to show an acceptable safety profile, mainly due to the function of the adult Wnt pathway in controlling tissue renewal and regeneration in the adult organs [4]. Therefore, the search for efficient and safe compounds targeting the Wnt pathway must go on.

Clofazimine is a riminophenazine derivative - a fully synthetic product based on a chemical scaffold initially obtained from lichens [5]. This drug was discovered in 1950s and approved as an essential part of the anti-leprosy complex treatment; however until today there is no consensus on the mechanism of its anti-bacterial activity [6, 7]. Reports on identification of clofazimine as an anticancer compound first appeared in mid-90s, however few mechanistic studies have been performed [8]. Those few identify clofazimine as an inhibitor of mitochondrial Kv1.3 channels [9], as a regulator of the PLA2induced cell death [10], or as an agent interfering with enzymes of the respiratory chain [11]. In our previous work, we reported identification of clofazimine as a novel inhibitor of Wnt signaling [12]. Taken together, these prior data point towards several mechanisms, which clofazimine might employ for suppression of cancer cell growth and induction of cell death.

Despite this multi-faceted activity profile, clofazimine is an agent with surprisingly few adverse effects: upon normal dosage it induces only grade 1-2 toxicities, and reaches higher grades only upon overdose [13]. This low toxicity profile creates significant hope for the repositioning of clofazimine as a targeted

Abbreviations: TNBC - triple-negative breast cancer; PLA2 - phospholipase A2; NSG - NOD-SCID-gamma; CDI coefficient of drug interaction 
therapy against numerous Wnt-dependent cancer types. It should be noted that the drug has already quite a track record of repositioning. Upon discovery, it was not approved for the treatment of tuberculosis, for which it had initially been developed, mostly due to lower efficacy and cosmetic defects upon application, and is currently approved only as a second-line therapy against the multidrug resistant tuberculosis [14]. Since leprosy is caused by another species of the Mycobacterium family, the drug was retested and further marketed as an agent against this disease [15]. In the beginning of 90's, anti-inflammatory properties of the compound were identified and, given its anti-microbial effect, the scope of applications of clofazimine was broadened to the treatment of skin infections caused by microorganisms [16]. Currently, clofazimine experiences a comeback as an anti-tuberculosis agent due to the rise of multidrug-resistant strains of this disease, which are still susceptible to clofazimine [17]. Much effort has been put in the compound development using modern medicinal chemistry approaches in order to reduce or completely eliminate its main side-effect - the skin discoloration [18-20].

The current work continues the exploration of the anti-Wnt properties of clofazimine and evaluates the preclinical anticancer efficacy and safety profiles of the drug in vitro and in vivo on a panel of TNBC (triple-negative breast cancer) cell lines and two xenograft models. Our results confirm that in TNBC, clofazimine acts through inhibition of Wnt signaling and that this activity mediates its growthinhibitory effects against a broad scope of TNBC tumors. Finally, we have investigated the interaction profile of clofazimine with a conventional cytotoxic agent doxorubicin in vitro and in vivo. Overall, these data pave the way for the clinical trials of clofazimine as a safe and efficient Wnt-targeting anticancer agent. 


\section{Materials and methods}

\subsection{Luciferase activity measurements}

The Wnt-induced and basal luciferase activity was analyzed as described [12, 21]. Briefly, 100 $\mu 1$ of BT20 cells, wild type or stably transfected with the TopFlash luciferase reporter [12] at 150000 cells/ml were distributed in a white opaque 96-well or 384-well plate. The cells were maintained in DMEM containing $10 \% \mathrm{FBS}$ and incubated at $37^{\circ} \mathrm{C}, 5 \% \mathrm{CO}_{2}$ overnight for attachment. Afterwards, the stably transfected BT-20 cells were additionally transfected with the plasmid constitutively (under the CMV promoter) expressing Renilla luciferase (Addgene, Cambridge, MA, USA), whereas wild-type cells were transfected by the 5:5:2 mixture of a reporter plasmid (Syngene), a Wnt5a-encoding or control plasmid and the Renilla luciferase plasmid. Transfection was carried out as described in the manufacturer's protocol using $12 \mu \mathrm{g} / \mathrm{ml}$ of DNA and $40 \mu \mathrm{l} / \mathrm{ml}$ XtremeGENE 9 reagent (Roche).

The next day, the medium of each well was replaced by $50 \mu 1$ (96-well plates) or $30 \mu 1$ (384-well plates) fresh medium containing Wnt3a (500ng/ml) (purified as described [22]) and clofazimine at concentrations indicated in the figures (following $1 \mathrm{~h}$ of pre-incubation with the compound). After overnight incubation, the supernatant in each well was removed and the luciferase activity was measured as described [12]. After 24h incubation, the cells were harvested and analyzed for activities of both firefly and Renilla luciferase as described [12, 21].

\subsection{Western blot for Wnt target genes}

Different TNBC and non-TNBC cell lines as indicated in the figures were seeded at 700'000 cells/well in 6-well plates. The next day, the medium was removed and replaced with a fresh medium pre-warmed to $37^{\circ} \mathrm{C}$ with or without clofazimine at $20 \mu \mathrm{M}$. After $1 \mathrm{~h}$ pre-incubation, if indicated, Wnt $3 \mathrm{a}$ was added at $200 \mathrm{ng} / \mathrm{ml}$. After $24 \mathrm{~h}$ incubation at $37^{\circ} \mathrm{C}$, the medium was removed, the cells in each well were washed twice with $500 \mu 1$ of $1 \mathrm{x}$ PBS and lysed directly in the well by addition of $100 \mu 1$ of ice-cold RIPA buffer (1x TBS, 4mM EDTA, 1\% Triton, 0.1\% SDS, 1x Protease inhibitor cocktail (Roche)) for 10min. The 
cells were re-suspended and the debris was removed by $10 \mathrm{~min}$ centrifugation at $16000 \mathrm{~g}, 4^{\circ} \mathrm{C}$. The supernatants were further analyzed by Western blot with antibodies against c-Myc (Abcam), Axin2 (Abcam) and $\alpha$-tubulin (Sigma) at 1:1000 dilution each.

\subsection{MTT assay}

$100 \mu \mathrm{l}$ of the indicated TNBC cell lines re-suspended at 30'000 cells/ml $\left(100^{\prime} 000 / \mathrm{ml}\right.$ for HCC-38 or $250^{\prime} 000 / \mathrm{ml}$ for IOWA-1T) were added into each well of a transparent 96-well plate. The cells were maintained in DMEM (DMEM/F12 in case of IOWA-1T) containing $10 \% \mathrm{FBS}$ and incubated at $37^{\circ} \mathrm{C}$, $5 \% \mathrm{CO}_{2}$ overnight. The medium of each well was replaced by $100 \mu \mathrm{l}$ fresh medium the next day containing the indicated concentrations of clofazimine. In case of combination experiments, the cells were first covered with $50 \mu \mathrm{l}$ of medium, and desired concentrations of clofazimine and doxorubicin in the well were created by adding them in $25 \mu \mathrm{l}$ of the corresponding pre-dilutions in the DMEM. After incubation for 3-4 days, the medium in each well was replaced by $100 \mu \mathrm{l}$ of $0.5 \mathrm{mg} / \mathrm{ml}$ Thiazolyl blue (Carl Roth) solution in 1xPBS. In case of IOWA-1T, which is a suspension cell line, $75 \mu \mathrm{l}$ of $1.5 \mathrm{mg} / \mathrm{ml}$ Thiazolyl blue solution was added directly in the $150 \mu \mathrm{l}$ of the medium. The plates were incubated for $3 \mathrm{~h}$ at $37^{\circ} \mathrm{C}$. Then the solution was removed and $50 \mu \mathrm{l}$ DMSO was added into each well. In case of IOWA-1T, the plates were centrifuged at $1000 \mathrm{~g}$ for $1 \mathrm{~min}$ and the supernatant was carefully removed prior to addition of DMSO. Absorbance at 570nm was measured in a plate reader (Victor3, PerkinElmer).

\subsection{Apoptosis and cell cycle assays}

Indicated cell lines were seeded at 500'000 per well in 6-well plates and incubated for $24 \mathrm{~h}$ in presence of $20 \mu \mathrm{M}$ clofazimine or DMSO. The cells were detached using the Accutase solution (BD) and stained for the corresponding assays. Apoptosis levels were measured using the Annexin V/PI staining kit (Sigma-Aldrich) according to the manufacturer's instructions. For cell cycle analysis, the cells were fixed in $70 \% \mathrm{EtOH}$ and stained with propidium iodide prior to analysis. For both assays, measurements 
were performed with the BD Galleos flow cytometer and the results were analyzed using the Flowing Software 2.5 (Turku University).

\subsection{Scratch-wound and colony formation assays with TNBC cells}

For migration analysis, the scratch-wound assay was used. Cells were seeded at 60'000 cells/well in 96-well flat-bottom plates. Next day, after attachment, the monolayer formed in each well was wounded in two places by a single strike of a $10 \mu \mathrm{l}$ pipette tip. The detached cells were removed by two washes with 1xPBS. For each experimental well, a random area of both scratches was labeled and a phase-contrast picture was taken. The cells were left for $24 \mathrm{~h}$ in presence or absence of $20 \mu \mathrm{M}$ clofazimine. Afterwards, pictures of the same area were taken and the migration of the cell front was analyzed by ImageJ.

For the colony formation assay, TNBC cells were seeded at 250 cells/well for HCC-1395, MDA-MB231 and HCC-38; 500/well for MBA-MB-468; 750/well for BT-20 and 1000/well for HCC-1806 in 6well plates. Next day, the medium was replaced with the one containing $20 \mu \mathrm{M}$ clofazimine. Colonies of 70-100 cells were formed after 8-14 days, were fixed by incubation in 4\% PFA in 1xPBS, pH 7.4 and visualized by staining with the crystal violet solution, and the number of colonies was counted.

\subsection{Mouse xenografts}

The experiments were approved by the Swiss Federal Veterinary Office and were carried out in accordance with the local animal welfare act. NOD-SCID-gamma (NSG) mice were injected intramammary with $50 \mu \mathrm{l}$ suspension of the following cells: $2 \times 10^{6}$ BT-20 cells stably transfected with pcDNA3-Luciferase construct (Addgene, Cambridge, MA, USA) or $5 \times 10^{5}$ IOWA-1T cells. For injection, the cells were detached by trypsin, washed 2x with ice-cold PBS and re-suspended in icecold Matrigel (BD, Franklin Lakes, NJ, USA, cat. \#356237). Tumor volume $\left(\mathrm{mm}^{3}\right)$ was determined using the following formula: tumor volume $=$ length $\times(\text { width })^{2} \times \pi / 6$. The drug treatment was started as soon as the tumor reached the volume of $\sim 100 \mathrm{~mm}^{3}$. 
For clofazimine treatment, the mice were separated into two groups: one receiving the drug and another receiving the vehicle $(\mathrm{N}=5$ each in case of IOWA-1T; $\mathrm{N}=3$ for vehicle, $\mathrm{N}=4$ for drug treatment in case of BT-20). Clofazimine $(2.5-10 \mathrm{mg} / \mathrm{ml}$, depending on the dose) was delivered by oral gavage as suspension in $10 \%$ Kolliphor, $1 \%$ CMC. The drug treatment group in all cases received the $200 \mathrm{mg} / \mathrm{kg}$ "loading dose" of clofazimine for 3 days in a row prior to switching to the doses delivered once in two days, as indicated in the figures and text. For the combination treatment, mice were separated into four groups: vehicle-treated $(\mathrm{N}=5)$, doxorubicin-treated $(\mathrm{N}=3)$, clofazimine-treated $(\mathrm{N}=4)$ and combinationtreated $(\mathrm{N}=4)$. These animals were treated by clofazimine or vehicle as described above and additionally the appropriate groups received $2 \mathrm{mg} / \mathrm{kg}$ once in 3 weeks injections of doxorubicin dissolved in $1 \times$ PBS at $0.5 \mathrm{mg} / \mathrm{ml}$.

In vivo imaging was performed using IVIS Lumina II (Xenogen). Mice were injected with 50mg/kg Dluciferin solution (Goldbio St. Louis, MO, USA) and luminescence was measured 10min postinjection. Intensity (expressed as photon flow) was quantified using the Living Image (PerkinElmer, Waltham, USA) software.

The coefficient of drug interaction (CDI) was calculated according to the following equation [23]: $\mathrm{CDI}=\mathrm{R} 12 /(\mathrm{R} 1 \times \mathrm{R} 2)$ where $\mathrm{R} 12$ is a tumor size in the combination-treated animals, and $\mathrm{R} 1$ and $\mathrm{R} 2$ are tumor sizes of the animals treated by each of the compounds alone.

\subsection{Slide preparation and immunofluorescent staining}

After removal from the animals, tumors were photographed, sliced into fragments of $\sim 300-400 \mathrm{~mm}^{3}$ and rinsed in the excess of ice-cold 1xPBS. Subsequently, they were fixed in fresh $10 \%$ PFA solution in 1xPBS for 3-5 days at $4^{\circ} \mathrm{C}$. The tumors were embedded in paraffin blocks and cut into slices $7-10 \mu \mathrm{m}$ thick. The slices were mounted on glass slides and stored at $4^{\circ} \mathrm{C}$. For the staining, the slides were first deparaffinized in three changes each of xylene, sequence of water mixtures of EtOH with decreasing concentrations $(95 \%, 70 \%$ and $50 \%)$ and finally water. Subsequently, antigen retrieval was performed 
in $20 \mathrm{mM}$ Tris-EDTA, $\mathrm{pH} 9.0$ with $0.1 \%$ Tween-20 by heating up the slides to $95^{\circ} \mathrm{C}$ for $20 \mathrm{~min}$ and gradual cooling to the room temperature. Further, the slides were blocked in $1 \times \mathrm{PBS} / 0.1 \%$ Triton X-100 with $3 \%$ of normal horse serum and stained with primary antibodies against $\beta$-catenin (BD Biosciences), MDR-1 (Sigma), Ki67 (BD Biosciences) at 1:200 dilution. Cleaved caspase-3 staining was performed using the respective antibodies (CST) at the 1:1'000 dilution and developed using the SuperBoost tyramide enhancer kit (Invitrogen). The slides were mounted using VectaSchield mounting medium and visualized using AiryScan LSM-880 confocal microscope (Zeiss). For scoring, each of the stained slides was assigned with the perceived level of membrane or cytoplasmic staining ranging from 0 (not observed) to 5 (maximum); the average score for all the stained tumors was calculated for presentation. 


\section{Results}

\subsection{Clofazimine is an efficient and specific inhibitor of the canonical Wnt signaling in a broad range of TNBC subtypes}

In our previous work [12], we demonstrated that clofazimine efficiently inhibited exogenous Wnt3ainduced signaling in HEK293T cells. Proliferation of the triple-negative breast cancer (TNBC) cell line BT-20, known to be dependent on the endogenous Wnt pathway activation [24], was inhibited with the similar $\mathrm{IC}_{50}$ of ca. $6 \mu \mathrm{M}$ [12]. Here, we first directly show that Wnt3a-induced signaling in BT-20 cells is inhibited with increasing concentrations of clofazimine, with the $\mathrm{IC}_{50}=7 \mu \mathrm{M}$, as measured with the TopFlash assay (Figure 1A). Noteworthy, acute cell toxicity measured by co-transfection with the Wntindependent transcriptional Renilla reporter could only be observed with clofazimine concentrations approaching $100 \mu \mathrm{M}$ (Figure 1B). As an additional readout of Wnt signaling in BT-20 cells, we monitored expression of Axin2 and c-Myc as two well-established [25-28] readouts of the Wnt signaling activity. Both endogenous and ectopic Wnt3a-induced levels of Axin2 and c-Myc were significantly suppressed by $20 \mu \mathrm{M}$ clofazimine (Figure 1C).

As TNBC is a heterogeneous disease, we next addressed whether the drug can reliably suppress Wnt signaling in a panel of TNBC cell lines. Additionally, non-TNBC breast cancer (BC) cell lines were also included in the analysis, since the Wnt pathway can be dysregulated also in other BC subtypes [26]. The panel we analyzed represented the clinical diversity of TNBC: three cell lines were luminallike ductal (BT-20), squamous (HCC-1806) and adeno-carcinoma (MDA-MB-468); one was basal-like adeno-carcinoma (MDA-MB-231); IOWA-1T represents TNBC stem cells population [29]; finally, HER2+ and ER/PR+ BCs were represented by MCF-7 and MDA-MB-453 cell lines, respectively. Clofazimine suppressed Axin2 in all the analyzed cell lines (Figure 1C-I). c-Myc was suppressed by clofazimine in most cell lines; however, in three of them (TNBC cell lines MDA-MB-468 and HCC1806, and ER/PR+ MDA-MB-453 cells) the levels of this protein remained intact (Figure 1E, G, I). 
We also ectopically stimulated the BC cell lines by purified Wnt3a. In response, Wnt target genes were found to be upregulated in most BC lines (Figure 1C-F). For these lines, we additionally verified the ability of clofazimine to suppress this induced response. For all these lines including MDA-MB-468 (where basal c-Myc levels were insensitive to the drug), clofazimine was able to decrease the response to purified Wnt3a (Figure 1C-F).

While the above readouts monitor the Wnt pathway at the level of target gene transcription, cytoplasmic and nuclear $\beta$-catenin represents a readout for more upstream levels of the pathway. TNBC cells have high endogenous levels of cytonuclear $\beta$-catenin through continuous activation of the Wnt pathway $[30,31]$. We thus analyzed the long-term effects of clofazimine on $\beta$-catenin levels in BT-20 cells, finding that $48 \mathrm{~h}$ drug treatment markedly decreased cytoplasmic $\beta$-catenin (from ca. $15 \%$ control cells positive for strong cytoplasmic $\beta$-catenin to ca. $2 \%$ upon clofazimine treatment) (Fig. 1J). In contrast, the membranic pool of $\beta$-catenin (mediating cell-cell contacts and not involved in the Wnt pathway [32]) remained relatively unaffected.

Taken together, these results indicate that clofazimine suppresses the canonical Wnt pathway (endogenous and exogenously induced), as measured by expression of Axin2, c-Myc, and TopFlash reporter genes/constructs in a broad panel of BC cell lines, as well as by monitoring cytoplasmic $\beta$ catenin levels. It appears that c-Myc expression in some cell lines is largely influenced by mechanisms additional to Wnt signaling [26].

We also accessed the specificity of clofazimine-induced inhibition of the canonical Wnt pathway using a set of luciferase reporters for other pathways (Supplementary Fig. 1). We found that in BT-20 cells, C/EBP and NFKB pathways were stimulated by Wnt5a - a non-canonical Wnt ligand [33] (Supplementary Fig. 1A and B), indicating that they may serve as the readouts of non-canonical Wnt pathway activity. Interaction between non-canonical Wnt pathway and MAPK pathway (one of which readouts is $\mathrm{C} / \mathrm{EBP})$ is well established $[34,35]$ and direct interaction of Wnt pathway with this transcriptional factor is crucial for adipocyte development [36, 37]. Both MAPK and NFkB pathways 
are promoted in non-canonical Wnt5a-dependent manner in dental pulp cells [38], while overall crosstalk between Wnt and NFאB is well described in various inflammatory models [39, 40]. However, clofazimine was unable to suppress activity of these pathways, both basal and Wnt5a-stimulated. Equally, clofazimine treatment exerted no effect in the panel of nine other pathway reporters (Supplementary Fig. 1C) indicating high specificity of the clofazimine inhibition towards canonical Wnt pathway.

\subsection{Clofazimine suppresses growth of a panel of TNBC cell lines through induction of apoptosis and cell cycle arrest}

We next moved to profile the anticancer properties of clofazimine against six TNBC cell lines (the cells used in Figure 1 above plus two additional basal-like ductal carcinoma cells, HCC-1395 and HCC-38) analyzing the drug's ability to suppress cell proliferation using the MTT assay. Concentrationdependent curves (Figure 2A, B) identified that $\mathrm{IC}_{50}$ of clofazimine against the TNBC lines lied within the $3-8 \mu \mathrm{M}$ interval agreeing well with its anti-Wnt $\mathrm{IC}_{50}$ (Figure $1 \mathrm{~A}$ ) and with the $\mathrm{IC}_{50}$ of growth suppression of BT-20 $(6 \mu \mathrm{M})$ we had reported earlier [12]. This proliferation arrest correlated well with the ability of clofazimine to suppress clonogenicity of a panel of TNBC cell lines (Figure 2C, D).

To analyze the mechanism of BC growth inhibition induced by clofazimine, we applied the Annexin V staining to assess the percentage of apoptotic and necrotic cells (Figure 2E), and propidium iodide staining to assess the cell cycle progression of the cells after clofazimine treatment (Figure 2F). The results indicate that clofazimine exerts a mixed mode of action to suppress proliferation of different BC lines, combining the induction of apoptosis with the G2/M-phase arrest.

In contrast to the clear anti-proliferative activity of the drug, clofazimine revealed only a limited effect on cancer cell motility as measured in the wound healing assay, partially suppressing motility of MDAMB-231 and MDA-MB-468 but no other TNBC cell lines at 10 $\mu \mathrm{M}$ (Figure $2 \mathrm{G}, \mathrm{H}$ ). This finding was 
expected, as tumor cell migration is known to depend less on the canonical Wnt pathway than other tumor characteristics [41].

\subsection{TNBC tumor xenograft growth is suppressed by oral clofazimine}

We continued our investigation by testing how oral regimen of clofazimine would affect growth of human TNBC cells grafted intramammary in NOD-SCID-gamma (NSG) mouse hosts. The drug is highly bioavailable and its allometric and pharmacokinetic properties are well described [42]. We therefore selected 50 and $100 \mathrm{mg} / \mathrm{kg}$ doses administered every other day since they corresponded to the maximal tested doses of clofazimine in humans (100-300 mg/day, [42, 43]). In agreement with the in vitro results described above, clofazimine delivery by oral gavage resulted in a profound suppression of the tumor growth initiated by IOWA-1T (Figure 3A-D) and BT-20 cells (Figure 3E-H) as estimated by electronic calipers during the trial run (Figure $3 \mathrm{~A}, \mathrm{~F})$ as well as tumor volume estimation after the animals' sacrifice (Figure 3C, D). Luminescence measurements from the tumors (BT-20 cells were stably transfected with constitutive firefly luciferase prior to xenografting) provided an additional readout (Figure 3E, H) confirming the manual assessment of the tumor volumes. Importantly, no adverse effects of 50 or $100 \mathrm{mg} / \mathrm{kg}$ clofazimine treatment were observed, as illustrated by the lack of body weight loss (Figure 3B, G) nor any other parameters (see below, Supplementary Tables 1 and 2). Moreover, our re-run of the trial with a decreased dose of clofazimine $(25 \mathrm{mg} / \mathrm{kg})$ yielded similar decrease of the tumor volume as was observed for 50 and $100 \mathrm{mg} / \mathrm{kg}$ dosage (Supplementary Fig. 2).

\subsection{On-target effect of clofazimine in vivo}

We further investigated whether the growth suppression of the xenografted tumor cells correlated with the inhibition of Wnt signaling in the tumor. To this end, we performed immunohistochemistry analysis of fixed paraffin slides of the tumors against two well established markers of Wnt signaling $-\beta$-catenin and its target gene MDR-1 [25, 26, 31, 44]. 
$\beta$-catenin revealed a striking change of the pattern of its intracellular distribution upon clofazimine treatment (Figure 4A). In control tumors, the vast majority of the cells displayed a strong cytoplasmic pattern correlating well with the high levels of activity of Wnt signaling in these cells in the growing tumor $[31,45]$. Upon clofazimine treatment, a dramatic increase was seen in the number of cells losing cytoplasmic $\beta$-catenin and exclusively showing the membrane $\beta$-catenin staining pattern, typical for low Wnt signaling levels, with the plasma membrane pool of $\beta$-catenin mediating Wnt-independent cell-cell contact function [45] (Figure 4A). To account for the heterogeneity of IOWA-1T xenograft tumors, we quantified the slides for the relative number of cells divided into four subtypes by the observed level of membrane and cytoplasmic $\beta$-catenin (Figure 4B) as well as performed slide scoring (Figure 4C) followed by statistical analysis. All these analyses clearly confirm the qualitative observations and reveal a picture of dramatic clofazimine-induced switch from the Wnt-on to the Wntoff status of the tumor cells. These in vivo findings correlate with the decrease of cytoplasmic $\beta$-catenin induced by clofazimine in cultured TNBC cells (Figure 1J above).

The second Wnt signaling marker, MDR-1, is a very attractive Wnt target gene due to its well-known function in the development of tumor drug resistance [46-48]. Upon visual inspection of the stainings, we observed a significant decrease in MDR-1 levels in the tumors after clofazimine treatment (Figure 4A). Since MDR-1 staining was not homogenous but appeared as staining-positive "zones", in order to quantify this decrease of MDR-1 staining, we counted these zones in the area of the same size between control and clofazimine-treated samples (Figure 4D). Such investigation revealed a clear quantifiable reduction in occurrence of such zones, along with their reduced brightness in clofazimine-treated samples.

We also confirmed that decreased growth in clofazimine-treated tumors is related to the pro-apoptotic and cell cycle-related effects of the clofazimine as we observed above in the in vitro experiments (Figure 2A). Indeed, immunofluorescent staining of the clofazimine-treated and control tumors for Ki67 and cleaved caspase-3 revealed that clofazimine-treated tumors have lower proportions of 
actively dividing cells and dramatically increased (around 5-fold) numbers of the apoptotic cleaved caspase-3-positive cells (Figure 4E and F).

\subsection{Clofazimine is compatible with the cytotoxic chemotherapy agent doxorubicin and this drug combination demonstrates additive effects in vitro and in vivo}

Depending on the exact diagnosis, targeted therapies are often combined with the reduced or even full regimens of the conventional cytotoxic agents [49-51]. It is therefore imperative to evaluate the effects of a drug interaction for any novel potential targeted agent. We thus further investigated the effects of clofazimine in the xenograft-bearing animals in combination with doxorubicin - a well-known chemotherapeutic agent and one of the first-line therapies for TNBC [52]. We used it at $2 \mathrm{mg} / \mathrm{kg}$ once in 3 weeks combined with clofazimine at $25 \mathrm{mg} / \mathrm{kg}$ daily, while the compounds alone, together with vehicle treatments, served as controls. The clofazimine+doxorubicin combination clearly and significantly exceeded the effects of either drugs when used alone (Figure 5A). Interestingly, while doxorubicin resulted in a significant systematic weight loss by the animals, the combination treatment did not aggravate this effect. In contrast, the combination rather tended to reduce this adverse effect of doxorubicin (Figure 5B). No other adverse reactions for control or treatment groups were identified during the study, which monitored parameters such as appearance of urine and feces, appearance of the skin, orifices, conscience and posture, see Supplementary Tables 1 and 2). Similarly, no overt organ damage was observed upon examination after sacrifice (data not shown). These data speak of an excellent in vivo compatibility of clofazimine with doxorubicin - both in terms of additive therapeutic effect and lack of aggravated adverse reaction.

The drug combination effect can be expressed quantitatively using various approaches. For the in vivo trial, we estimated the coefficient of drug interaction (CDI, [23], see materials and methods) for each day of the trial. This simple coefficient is useful when it is difficult to perform a dose-dependent study and shows values from zero to one if the effect of the two compound is synergistic, and above one in 
the case of antagonism. As demonstrated on Figure 5C, when calculated for each time point, the CDI fluctuated around one starting from the very beginning of the measurements, and averaged at 1.06 over the whole period of treatment. This is a clear indicative of additivity of these compounds on the tumor growth.

We decided to investigate this effect in more details in vitro using our panel of TNBC cell lines. We employed a comprehensive 2D approach for the drug combination analysis, assessing drug interactions over the complete range of effective doses for the two drugs. It was followed by Loewe modeling for estimation of the drug combination effect using the Combenefit software [53]. The main readout of this analysis is called the synergism/antagonism matrix where for each combination point, the coefficient ranges from -100 (the strongest antagonism) to +100 (the strongest synergism) and is coded in the color other than light green in case if the value passes statistical significance test (Supplementary Figure 3). As can be seen from representative examples of these matrices for different TNBC cell lines (Supplementary Figure 3), paralleling the in vivo results, this coefficient fluctuates around zero for most of concentration ranges across the TNBC panel, indicating predominance of the additive effect. The magnitude of any observed synergistic or antagonistic effects is small and may be found only in a narrow concentration range (Supplementary Figure 3 and 4).

Quantitatively, this is summarized on Figure 5D, where we used the weighted summary synergism and antagonism values calculated for such matrix from each independent repeat. It is evident that for all the cell lines, areas of weak synergism exist (along with areas of weak antagonism). The overall score of the synergistic effect is within the range of 5-15, which means that broadly the interaction between clofazimine and doxorubicin can be considered additive rather than synergistic.

We can therefore summarize that clofazimine and doxorubicin are compatible in vivo and in vitro, and that their anticancer effect is overall additive, with concomitant absence of additivity of adverse effects. Cumulatively, these findings lay the ground for the clinical development of clofazimine as targeted anticancer therapy, to be potentially applied in combination with conventional cytotoxic therapies. 


\section{Discussion}

The ability of clofazimine to inhibit growth of cancer cells in vitro was first discovered and evaluated in mid-90s $[10,11,54,55]$. However, these studies were typically not supported by mechanistic investigation, impeding subsequent drug development. Thus, anti-cancer clinical trials of clofazimine were small-scale, largely inconclusive and did not have a continuation $[54,56]$. Our re-discovery of clofazimine as an anticancer agent with a clear mechanism of action within the Wnt pathway [12] provided new hope for application of this compound and its derivatives in oncology. Our current work directs these prior findings to one of the most troublesome and deadly cancers - triple-negative breast cancer (TNBC) - which is known to depend on the activity of the Wnt pathway [26, 57]. We selected a panel of TNBC cell lines representing major clinically relevant subtypes (luminal-like, basal-like) as well as tumor-initiating cells ("cancer stem cells"), which are responsible for the resistance of this disease to chemotherapy [58]. We profiled the activity of clofazimine, both in terms of inhibition of the Wnt pathway and inhibition of cell proliferation, against this panel, revealing the strong promise of the drug against various TNBC lines including the tumor-initiating cells.

These data, taken together with the well-established safety profile of clofazimine, prompted us to confirm its activity using in vivo models. We chose BT-20 as a somatic cancer cell line with a proven track of Wnt-dependent growth [24, 59], and the IOWA-1T line corresponding to the chemoresistant cancer progenitor population [29], which has also been described to have and depend on high levels of Wnt signaling $[60,61]$. The second line was also our obvious choice for combination treatment, since it is particularly relevant for chemoresisitant tumors.

The dosage was adjusted based on the known data on clofazimine dose/plasma levels and tissue accumulation, as well as allometric scaling described for the drug [42, 43]. Thus, 50mg/kg of oral clofazimine given every other day in mice corresponded to about 100-300 $\mathrm{mg}$ per day of the human dose and expected to result in around $50-100 \mu \mathrm{M}$ of tissue levels (up to $500 \mu \mathrm{M}$ in some tissues) which 
is well above its typical in vitro $\mathrm{IC}_{50}$ of $3-8 \mu \mathrm{M}$. It should be noted that lipophilic clofazimine has an extreme propensity to accumulate in tissues, while its blood plasma levels remain flat at around $1 \mu \mathrm{M}$ at the doses above around $10 \mathrm{mg} / \mathrm{kg}$ [42]. Correspondingly, clofazimine demonstrated a profound ability to inhibit tumor growth in the two xenograft models we studied, including highly aggressive and fastgrowing tumor derived from the IOWA-1T cell line, which is highly resistant to a conventional antiTNBC therapy doxorubicin in vivo (Figure 5A, [29]). In addition to this anti-cancer effect, we observed no overt adverse reactions in the animals. The anti-tumor effect, in contrast, was observed even at the low doses $(25 \mathrm{mg} / \mathrm{kg})$ indicating potentially a very broad therapeutic window for the compound.

Despite many investigations of clofazimine action on cultured cells, only a few studies dealt with molecular understanding of the target and the mode of action of the drug. One of such line of studies highlighted the potassium channel Kv1.3 as a target of clofazimine, with inhibition of the channel by the drug inducing cancer cell apoptosis through mitochondrial ROS production $[9,62]$ in preclinical models of chronic lymphocytic leukemia [9], melanoma [62] and pancreatic adenocarcinoma [63]. Inhibitors of Kv1.3 are known to be immunosuppressive, and such properties of clofazimine have been described even prior to the discovery of its ability to inhibit Kv1.3 [7, 16]. Among the immune cells, clofazimine preferentially targets T-cells through Kv1.3 and macrophages through formation of biocrystals $[64,65]$. These inhibitions might produce conflicting effects on the anti-tumor immunity, as the tumor-infiltrating lymphocytes exert anti-tumor activities [66], while tumor-associated macrophages are generally considered to be immunosuppressive and are associated with poor clinical outcome [67]. The respectful role of the molecular target of clofazimine (Kv1.3 vs. the Wnt pathway vs. both at once) in different tumor types, as well of the role of clofazimine-mediated inhibition of one or both of these targets in different types of tumor-associated immune cells, awaits further investigation. It is worth mentioning in this regard that activation the tumor Wnt pathway promotes evasion from the immune control through enhanced PD-L1 expression in TNBC [68]. 
Focusing on Wnt signaling in TNBC, we identified a profound on-target effect of clofazimine in the animal models. The drug blocked $\beta$-catenin accumulation in the cytoplasm of tumor cells, representing a switch from a Wnt-on to the Wnt-off state. Additionally, reduction in a Wnt target gene MDR-1 was observed upon clofazimine treatment. Apart from being and additional confirmation of clofazimine ontarget effect in vivo, this finding provides a mechanistic explanation to the phenomenon of MDR-1related chemosensitization observed for clofazimine at the dawn of studies of its anti-cancer activity $[55,69]$. We further report here the investigation of combination of clofazimine with doxorubicin, one of the most frequently used treatments for TNBC. The two drugs largely reveal additive anti-tumor effects in vitro and in vivo, without additivity for the adverse effects, agreeing with the prior clinical trial on combining clofazimine with doxorubicin, which demonstrated no additional toxicity [56].

Overall, our work paints clofazimine as a highly promising targeted drug against TNBC and other Wntdependent tumors. Decades of application of the drug to patients with infectious diseases have established a thorough PK profile with detailed description of the expectable moderate side effects. The novel mechanism of action of the drug and its additivity with a conventional chemotherapy permits initiation of repositioning clinical trials. In case of success, this work will bring about first targeted therapy against the deadliest form of breast cancer. 
Animal experimentation: The experiments performed in current paper were approved and done under the license of Swiss Federal Veterinary Office.

Competing interests: The authors declare that they have no competing interests

Funding: This work was supported by a grant from the Swiss Cancer League (grant number KFS2978-08-2012), a grant from Novartis Foundation for Medical-Biological Research (grant number 17C153), and a grant from Stiftung zur Krebsbekämpfung (grant number 397) to V.L.K.

Authors' contributions: AK and KA performed and co-performed most of the experiments; JX performed most of the experiments indicated on Fig. 2. AB contributed at the revision stage of the paper. VLK designed and organized the work, AK and VLK wrote the paper; all the authors contributed to finalizing the paper. 


\section{References.}

[1] H. Clevers, R. Nusse, Wnt/beta-catenin signaling and disease, Cell, 149 (2012) 1192-1205.

[2] R. Nusse, H. Clevers, Wnt/beta-Catenin Signaling, Disease, and Emerging Therapeutic Modalities, Cell, 169 (2017) 985-999.

[3] A. Blagodatski, D. Poteryaev, V. Katanaev, Targeting the Wnt pathways for therapies, Molecular and Cellular Therapies, 2 (2014) 28.

[4] M. Kahn, Can we safely target the WNT pathway?, Nat Rev Drug Discov, 13 (2014) 513-532.

[5] V.M. Reddy, J.F. O'Sullivan, P.R. Gangadharam, Antimycobacterial activities of riminophenazines, The Journal of antimicrobial chemotherapy, 43 (1999) 615-623.

[6] T. Yano, S. Kassovska-Bratinova, J.S. Teh, J. Winkler, K. Sullivan, A. Isaacs, N.M. Schechter, H. Rubin, Reduction of clofazimine by mycobacterial type $2 \mathrm{NADH}$ :quinone oxidoreductase: a pathway for the generation of bactericidal levels of reactive oxygen species, The Journal of biological chemistry, 286 (2011) 10276-10287.

[7] J.L. Arbiser, S.L. Moschella, Clofazimine: a review of its medical uses and mechanisms of action, Journal of the American Academy of Dermatology, 32 (1995) 241-247.

[8] W. Levis, T. Rendini, Clofazimine Mechanisms of Action in Mycobacteria, HIV, and Cancer, The Journal of infectious diseases, 215 (2017) 1488.

[9] L. Leanza, L. Trentin, K.A. Becker, F. Frezzato, M. Zoratti, G. Semenzato, E. Gulbins, I. Szabo, Clofazimine, Psora-4 and PAP-1, inhibitors of the potassium channel Kv1.3, as a new and selective therapeutic strategy in chronic lymphocytic leukemia, Leukemia, 27 (2013) 1782-1785.

[10] C.E. Van Rensburg, A.M. Van Staden, R. Anderson, The riminophenazine agents clofazimine and B669 inhibit the proliferation of cancer cell lines in vitro by phospholipase A2-mediated oxidative and nonoxidative mechanisms, Cancer research, 53 (1993) 318-323.

[11] R.M. Sri-Pathmanathan, J.A. Plumb, K.C. Fearon, Clofazimine alters the energy metabolism and inhibits the growth rate of a human lung-cancer cell line in vitro and in vivo, International journal of cancer, 56 (1994) 900-905.

[12] A.V. Koval, P. Vlasov, P. Shichkova, S. Khunderyakova, Y. Markov, J. Panchenko, A. Volodina, F.A. Kondrashov, V.L. Katanaev, Anti-leprosy drug clofazimine inhibits growth of triple-negative breast cancer cells via inhibition of canonical Wnt signaling, Biochem Pharmacol, 87 (2014) 571-578. [13] C.E. Van Rensburg, R. Anderson, J.F. O'Sullivan, Riminophenazine compounds: pharmacology and anti-neoplastic potential, Critical reviews in oncology/hematology, 25 (1997) 55-67.

[14] J.H. Grosset, S. Tyagi, D.V. Almeida, P.J. Converse, S.Y. Li, N.C. Ammerman, W.R. Bishai, D. Enarson, A. Trebucq, Assessment of clofazimine activity in a second-line regimen for tuberculosis in mice, American journal of respiratory and critical care medicine, 188 (2013) 608-612.

[15] J.C. Garrelts, Clofazimine: a review of its use in leprosy and Mycobacterium avium complex infection, DICP : the annals of pharmacotherapy, 25 (1991) 525-531.

[16] P. Gurfinkel, J.C. Pina, M. Ramos-e-Silva, Use of clofazimine in dermatology, Journal of drugs in dermatology: JDD, 8 (2009) 846-851.

[17] B. Lechartier, S.T. Cole, Mode of Action of Clofazimine and Combination Therapy with Benzothiazinones against Mycobacterium tuberculosis, Antimicrobial agents and chemotherapy, 59 (2015) 4457-4463.

[18] D. Zhang, Y. Lu, K. Liu, B. Liu, J. Wang, G. Zhang, H. Zhang, Y. Liu, B. Wang, M. Zheng, L. Fu, Y. Hou, N. Gong, Y. Lv, C. Li, C.B. Cooper, A.M. Upton, D. Yin, Z. Ma, H. Huang, Identification of less lipophilic riminophenazine derivatives for the treatment of drug-resistant tuberculosis, J Med Chem, 55 (2012) 8409-8417.

[19] M. Tonelli, F. Novelli, B. Tasso, A. Sparatore, V. Boido, F. Sparatore, S. Cannas, P. Molicotti, S. Zanetti, S. Parapini, R. Loddo, Antitubercular activity of 
quinolizidinyl/pyrrolizidinylalkyliminophenazines, Bioorganic \& medicinal chemistry, 22 (2014) 68376845.

[20] A. Barteselli, M. Casagrande, N. Basilico, S. Parapini, C.M. Rusconi, M. Tonelli, V. Boido, D. Taramelli, F. Sparatore, A. Sparatore, Clofazimine analogs with antileishmanial and antiplasmodial activity, Bioorganic \& medicinal chemistry, 23 (2015) 55-65.

[21] H.V. Shaw, A. Koval, V.L. Katanaev, A high-throughput assay pipeline for specific targeting of frizzled GPCRs in cancer, Methods Cell Biol, 149 (2019) 57-75.

[22] A. Koval, V.L. Katanaev, Wnt3a stimulation elicits G-protein-coupled receptor properties of mammalian Frizzled proteins, Biochem J, 433 (2011) 435-440.

[23] X. Li, Z. Lin, B. Zhang, L. Guo, S. Liu, H. Li, J. Zhang, Q. Ye, beta-elemene sensitizes hepatocellular carcinoma cells to oxaliplatin by preventing oxaliplatin-induced degradation of copper transporter 1, Scientific reports, 6 (2016) 21010.

[24] L. Yang, X. Wu, Y. Wang, K. Zhang, J. Wu, Y.C. Yuan, X. Deng, L. Chen, C.C. Kim, S. Lau, G. Somlo, Y. Yen, FZD7 has a critical role in cell proliferation in triple negative breast cancer, Oncogene, 30 (2011) 4437-4446.

[25] A.B. Ramakrishnan, K.M. Cadigan, Wnt target genes and where to find them, F1000Research, 6 (2017) 746.

[26] A. Koval, V.L. Katanaev, Dramatic dysbalancing of the Wnt pathway in breast cancers, Sci Rep, 8 (2018) 7329.

[27] T.C. He, A.B. Sparks, C. Rago, H. Hermeking, L. Zawel, L.T. da Costa, P.J. Morin, B. Vogelstein, K.W. Kinzler, Identification of c-MYC as a target of the APC pathway, Science, 281 (1998) 1509-1512. [28] E.H. Jho, T. Zhang, C. Domon, C.K. Joo, J.N. Freund, F. Costantini, Wnt/beta-catenin/Tcf signaling induces the transcription of Axin2, a negative regulator of the signaling pathway, Mol Cell Biol, 22 (2002) 1172-1183.

[29] M.V. Bogachek, J.M. Park, J.P. De Andrade, M.V. Kulak, J.R. White, T. Wu, P.M. Spanheimer, T.B. Bair, A.K. Olivier, R.J. Weigel, A novel animal model for locally advanced breast cancer, Annals of surgical oncology, 22 (2015) 866-873.

[30] W.-C. Chen, Y.-A. Lai, Y.-C. Lin, J.-W. Ma, L.-F. Huang, N.-S. Yang, C.-T. Ho, S.-C. Kuo, T.-D. Way, Curcumin Suppresses Doxorubicin-Induced Epithelial-Mesenchymal Transition via the Inhibition of TGF- $\beta$ and PI3K/AKT Signaling Pathways in Triple-Negative Breast Cancer Cells, Journal of Agricultural and Food Chemistry, 61 (2013) 11817-11824.

[31] A.I. Khramtsov, G.F. Khramtsova, M. Tretiakova, D. Huo, O.I. Olopade, K.H. Goss, Wnt/betacatenin pathway activation is enriched in basal-like breast cancers and predicts poor outcome, Am J Pathol, 176 (2010) 2911-2920.

[32] T. Valenta, G. Hausmann, K. Basler, The many faces and functions of beta-catenin, EMBO J, 31 (2012) 2714-2736.

[33] M. Endo, M. Nishita, M. Fujii, Y. Minami, Insight into the role of Wnt5a-induced signaling in normal and cancer cells, Int Rev Cell Mol Biol, 314 (2015) 117-148.

[34] R.K. Bikkavilli, C.C. Malbon, Mitogen-activated protein kinases and Wnt/beta-catenin signaling: Molecular conversations among signaling pathways, Communicative \& integrative biology, 2 (2009) 46-49.

[35] Y. Zhang, T. Pizzute, M. Pei, A review of crosstalk between MAPK and Wnt signals and its impact on cartilage regeneration, Cell and tissue research, 358 (2014) 633-649.

[36] S.E. Ross, N. Hemati, K.A. Longo, C.N. Bennett, P.C. Lucas, R.L. Erickson, O.A. MacDougald, Inhibition of adipogenesis by Wnt signaling, Science, 289 (2000) 950-953.

[37] S. Chirumbolo, G. Bjorklund, Can Wnt5a and Wnt non-canonical pathways really mediate adipocyte de-differentiation in a tumour microenvironment?, European journal of cancer, 64 (2016) 96100. 
[38] Y. Zhao, C.L. Wang, R.M. Li, T.Q. Hui, Y.Y. Su, Q. Yuan, X.D. Zhou, L. Ye, Wnt5a promotes inflammatory responses via nuclear factor kappaB (NF-kappaB) and mitogen-activated protein kinase (MAPK) pathways in human dental pulp cells, The Journal of biological chemistry, 289 (2014) 2102821039.

[39] T. Fukuda, L. Chen, T. Endo, L. Tang, D. Lu, J.E. Castro, G.F. Widhopf, 2nd, L.Z. Rassenti, M.J. Cantwell, C.E. Prussak, D.A. Carson, T.J. Kipps, Antisera induced by infusions of autologous AdCD154-leukemia B cells identify ROR1 as an oncofetal antigen and receptor for Wnt5a, Proceedings of the National Academy of Sciences of the United States of America, 105 (2008) 3047-3052.

[40] B. Ma, M.O. Hottiger, Crosstalk between Wnt/beta-Catenin and NF-kappaB Signaling Pathway during Inflammation, Frontiers in immunology, 7 (2016) 378.

[41] A.H. Nwabo Kamdje, P. Takam Kamga, R. Tagne Simo, L. Vecchio, P.F. Seke Etet, J.M. Muller, G. Bassi, E. Lukong, R. Kumar Goel, J. Mbo Amvene, M. Krampera, Developmental pathways associated with cancer metastasis: Notch, Wnt, and Hedgehog, Cancer biology \& medicine, 14 (2017) 109-120.

[42] R.V. Swanson, J. Adamson, C. Moodley, B. Ngcobo, N.C. Ammerman, A. Dorasamy, S. Moodley, Z. Mgaga, A. Tapley, L.A. Bester, S. Singh, J.H. Grosset, D.V. Almeida, Pharmacokinetics and pharmacodynamics of clofazimine in a mouse model of tuberculosis, Antimicrobial agents and chemotherapy, 59 (2015) 3042-3051.

[43] D.K. Banerjee, G.A. Ellard, P.T. Gammon, M.F. Waters, Some observations on the pharmacology of clofazimine (B663), The American journal of tropical medicine and hygiene, 23 (1974) 1110-1115.

[44] M. Flahaut, R. Meier, A. Coulon, K.A. Nardou, F.K. Niggli, D. Martinet, J.S. Beckmann, J.M. Joseph, A. Muhlethaler-Mottet, N. Gross, The Wnt receptor FZD1 mediates chemoresistance in neuroblastoma through activation of the Wnt/beta-catenin pathway, Oncogene, 28 (2009) 2245-2256.

[45] A. Serafino, G. Sferrazza, A. Colini Baldeschi, G. Nicotera, F. Andreola, E. Pittaluga, P. Pierimarchi, Developing drugs that target the Wnt pathway: recent approaches in cancer and neurodegenerative diseases, Expert Opin Drug Discov, 12 (2017) 169-186.

[46] D.Y. Shen, W. Zhang, X. Zeng, C.Q. Liu, Inhibition of Wnt/beta-catenin signaling downregulates P-glycoprotein and reverses multi-drug resistance of cholangiocarcinoma, Cancer science, 104 (2013) 1303-1308.

[47] Y.H. Wang, Y. Imai, M. Shiseki, J. Tanaka, T. Motoji, Knockdown of the Wnt receptor Frizzled-1 (FZD1) reduces MDR1/P-glycoprotein expression in multidrug resistant leukemic cells and inhibits leukemic cell proliferation, Leukemia research, 67 (2018) 99-108.

[48] Z.M. Zhang, J.F. Wu, Q.C. Luo, Q.F. Liu, Q.W. Wu, G.D. Ye, H.Q. She, B.A. Li, Pygo2 activates MDR1 expression and mediates chemoresistance in breast cancer via the Wnt/beta-catenin pathway, Oncogene, 35 (2016) 4787-4797.

[49] A. Simon, H.R. Kourie, J. Kerger, Is there still a role for cytotoxic chemotherapy after targeted therapy and immunotherapy in metastatic melanoma? A case report and literature review, Chinese journal of cancer, 36 (2017) 10.

[50] D.H. Johnson, Targeted therapies in combination with chemotherapy in non-small cell lung cancer, Clinical cancer research : an official journal of the American Association for Cancer Research, 12 (2006) 4451s-4457s.

[51] P.A. Bunn, Jr., Triplet combination chemotherapy and targeted therapy regimens, Oncology, 15 (2001) 26-32.

[52] B.S. Yadav, S.C. Sharma, P. Chanana, S. Jhamb, Systemic treatment strategies for triple-negative breast cancer, World J Clin Oncol, 5 (2014) 125-133.

[53] G.Y. Di Veroli, C. Fornari, D. Wang, S. Mollard, J.L. Bramhall, F.M. Richards, D.I. Jodrell, Combenefit: an interactive platform for the analysis and visualization of drug combinations, Bioinformatics, 32 (2016) 2866-2868. 
[54] P. Ruff, M.R. Chasen, J.E. Long, C.E. van Rensburg, A phase II study of oral clofazimine in unresectable and metastatic hepatocellular carcinoma, Annals of oncology : official journal of the European Society for Medical Oncology, 9 (1998) 217-219.

[55] C.E. van Rensburg, G.K. Joone, J.F. O'Sullivan, Clofazimine and B4121 sensitize an intrinsically resistant human colon cancer cell line to P-glycoprotein substrates, Oncology reports, 7 (2000) 193195.

[56] C.I. Falkson, G. Falkson, A phase II evaluation of clofazimine plus doxorubicin in advanced, unresectable primary hepatocellular carcinoma, Oncology, 57 (1999) 232-235.

[57] S.G. Pohl, N. Brook, M. Agostino, F. Arfuso, A.P. Kumar, A. Dharmarajan, Wnt signaling in triplenegative breast cancer, Oncogenesis, 6 (2017) e310.

[58] A. Pavlopoulou, Y. Oktay, K. Vougas, M. Louka, C.E. Vorgias, A.G. Georgakilas, Determinants of resistance to chemotherapy and ionizing radiation in breast cancer stem cells, Cancer Lett, 380 (2016) 485-493.

[59] P. De, J.H. Carlson, H. Wu, A. Marcus, B. Leyland-Jones, N. Dey, Wnt-beta-catenin pathway signals metastasis-associated tumor cell phenotypes in triple negative breast cancers, Oncotarget, 7 (2016) 43124-43149.

[60] M. Katoh, Canonical and non-canonical WNT signaling in cancer stem cells and their niches: Cellular heterogeneity, omics reprogramming, targeted therapy and tumor plasticity (Review), International journal of oncology, 51 (2017) 1357-1369.

[61] K.C. Valkenburg, C.R. Graveel, C.R. Zylstra-Diegel, Z. Zhong, B.O. Williams, Wnt/beta-catenin Signaling in Normal and Cancer Stem Cells, Cancers, 3 (2011) 2050-2079.

[62] L. Leanza, B. Henry, N. Sassi, M. Zoratti, K.G. Chandy, E. Gulbins, I. Szabo, Inhibitors of mitochondrial Kv1.3 channels induce Bax/Bak-independent death of cancer cells, EMBO molecular medicine, 4 (2012) 577-593.

[63] A. Zaccagnino, A. Manago, L. Leanza, A. Gontarewitz, B. Linder, M. Azzolini, L. Biasutto, M. Zoratti, R. Peruzzo, K. Legler, A. Trauzold, H. Kalthoff, I. Szabo, Tumor-reducing effect of the clinically used drug clofazimine in a SCID mouse model of pancreatic ductal adenocarcinoma, Oncotarget, 8 (2017) 38276-38293.

[64] G.S. Yoon, R.K. Keswani, S. Sud, P.M. Rzeczycki, M.D. Murashov, T.A. Koehn, T.J. Standiford, K.A. Stringer, G.R. Rosania, Clofazimine Biocrystal Accumulation in Macrophages Upregulates Interleukin 1 Receptor Antagonist Production To Induce a Systemic Anti-Inflammatory State, Antimicrobial agents and chemotherapy, 60 (2016) 3470-3479.

[65] Y.R. Ren, F. Pan, S. Parvez, A. Fleig, C.R. Chong, J. Xu, Y. Dang, J. Zhang, H. Jiang, R. Penner, J.O. Liu, Clofazimine inhibits human Kv1.3 potassium channel by perturbing calcium oscillation in $\mathrm{T}$ lymphocytes, PloS one, 3 (2008) e4009.

[66] L. Conforti, Potassium channels of T lymphocytes take center stage in the fight against cancer, Journal for immunotherapy of cancer, 5 (2017) 2.

[67] T. Chanmee, P. Ontong, K. Konno, N. Itano, Tumor-associated macrophages as major players in the tumor microenvironment, Cancers, 6 (2014) 1670-1690.

[68] L. Castagnoli, V. Cancila, S.L. Cordoba-Romero, S. Faraci, G. Talarico, B. Belmonte, M.V. Iorio, M. Milani, T. Volpari, C. Chiodoni, A. Hidalgo-Miranda, E. Tagliabue, C. Tripodo, S. Sangaletti, M. Di Nicola, S.M. Pupa, WNT signaling modulates PD-L1 expression in the stem cell compartment of triplenegative breast cancer, Oncogene, (2019).

[69] M.S. Myer, C.E. Van Rensburg, Chemosensitizing interactions of clofazimine and B669 with human K562 erythroleukaemia cells with varying levels of expression of P-glycoprotein, Cancer Lett, 99 (1996) 73-78. 


\section{Figure legends}

Figure 1. Wnt signaling is suppressed in various TNBC and non-TNBC cell lines $(A, B)$ Dosedependent inhibition of the Wnt3a-induced response in BT-20 TNBC cells transfected with the Wntdependent TopFlash reporter construct (A) and with the Wnt-independent CMV-Renilla reporter construct (B). (C-F) Endogenous and Wnt3a-induced expression levels of the Wnt target genes Axin2 and c-Myc are reduced in a panel of BC cell lines. Representative Western blots are shown on the left of each panel, and quantification of expression - on the right. (G-I) In three BC lines, ectopic Wnt3a did not increase Axin2 and c-Myc levels, yet the endogenous levels of these target genes were sensitive to clofazimine. $(\mathbf{J})$ Immunofluorescent staining of $\beta$-catenin in BT-20 cells treated with clofazimine for 48h reveals a dramatic decrease in the cells positive for cytoplasmic $\beta$-catenin (marked with arrows); quantification is provided on the right.

Figure 2. Treatement with clofazimine reduces cancer hallmarks and induces apoptosis and cell cycle arrest $(\mathrm{A}, \mathrm{B})$ Dose-dependent survival curves $(\mathrm{A})$ and $\mathrm{IC}_{50}$ values $(\mathrm{B})$ of clofazimine obtained for a panel of six TNBC cell lines. (C,D) Clofazimine $(20 \mu \mathrm{M})$ potently suppresses colony formation by the TNBC cell lines; MDA-MB-468 cells are shown as example (D). (E,F) Analysis of apoptosis and cell cycle progression in the $20 \mu \mathrm{M}$ clofazimine-treated cell lines demonstrate that the drug uses both these mechanisms to exert its effect on tumor cells. Data are provided as percentage to the vehicle-treated cells. (G,H) Scratch-wound assay indicates that clofazimine at $20 \mu \mathrm{M}$ has little or no effect on the migration ability of TNBC cells. Only two cell lines, MDA-MB-231 and MDA-MB-468, were sensitive to the drug; MDA-MB-468 cells are shown as example $(\mathrm{H})$.

Figure 3. TNBC xenograft tumor growth is suppressed by oral clofazimine (A-D) Treatment of intramammary IOWA-1T tumor xenograft-bearing NGS mice results in significant suppression of tumor growth at both 100 and $50 \mathrm{mg} / \mathrm{kg}$ daily oral dose. On (A) the graph shows tumor volume 
estimated by electronic calipers. Body weight (B) remains stable across the experiment. (C) shows the tumors extracted from the animals by the end of the trial and (D) - estimation of their volume. (E-H) Effects of clofazimine on growth of tumor xenografts obtained by orthotopic injections of BT-20 cells expressing firefly luciferase. (E) shows total luminescence of the tumor as the tumor size readout; (F) shows the parallel measurements of the tumor volume using electronic calipers. $(\mathrm{G})$ demonstrates that the body weight of the animals was stable across the experiment. (H) shows tumor luminescence in control and clofazimine-treated animals on the 3rd week of treatment.

Figure 4. Clofazimine elicits on-target anti-Wnt effects in TNBC xenograft tumors (A) Immunofluorescent stainings of $\beta$-catenin and MDR-1 reveal a dramatic effect of clofazimine on the Wnt signaling in tumor xenografts: MDR-1 levels are reduced, $\beta$-catenin switches from cytoplasmic to membrane localization. (B) Quantification of the effects of clofazimine on $\beta$-catenin localization: each cell from the section above was assigned to one of the four types depending on the observed pattern of $\beta$-catenin localization. Relative cell counts reveal that clofazimine treatment significantly enriches the proportion of cells with high membrane and low cytoplasmic $\beta$-catenin, while correspondingly decreasing the share of the cells positive for the cytoplasmic staining only. (C) Similar results were obtained using the scoring of the slides by the observed levels of cytoplasmic and membrane $\beta$-catenin. (D) Since MDR-1 staining is not homogenous but occurs in the form of "positive areas", we quantified the number of these areas per slide. This quantification demonstrates a significant decrease in the number of such areas in clofazimine-treated tumors. (E, F) Immunofluorescent stainings of the tumor sections for Ki67 and cleaved caspase-3 and their quantifications demonstrate a significant decrease in the proportion of proliferating cells in the clofazimine-treated tumor along with 5-fold elevated levels of apoptosis. 
Figure 5. Clofazimine is compatible with cytotoxic chemotherapy producing additive effects (A) Treatment of the xenograft-bearing animals with clofazimine in combination with doxorubicin exerts a statistically significant decrease in tumor growth as compared to either doxorubicin or clofazimine as single agents. (B) Such combination did not affect the body weight of the animals. (C) Coefficient of drug interaction (CDI) was calculated for each day of the measurement and average of it is presented on the graph. The data demonstrate additivity of the clofazimine and doxorubicin effects in vivo. (D) Summary graph showing means of weighted average values of synergism and antagonism obtained from independent repeats of in vitro 2D drug interaction analysis on a panel of TNBC cell lines. Combenefit software analysis matrices and the representative experiments are shown in Supplementary Fig. 3 and 4. 
A

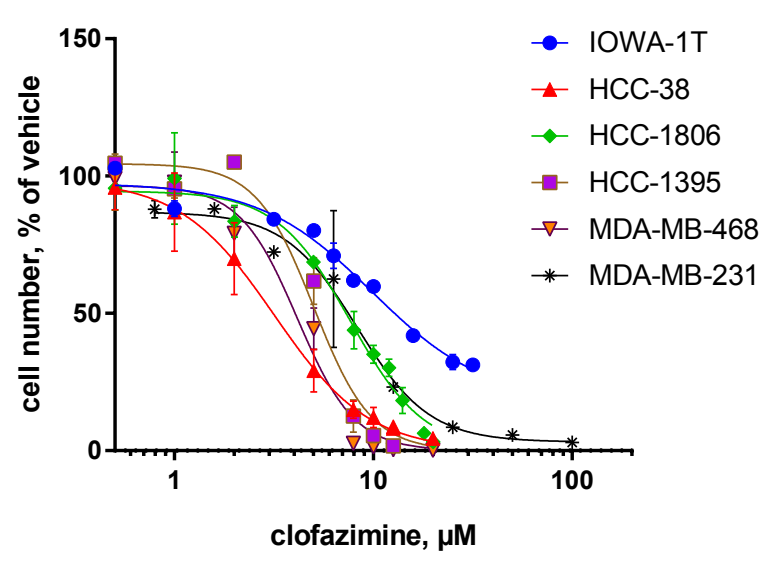

C
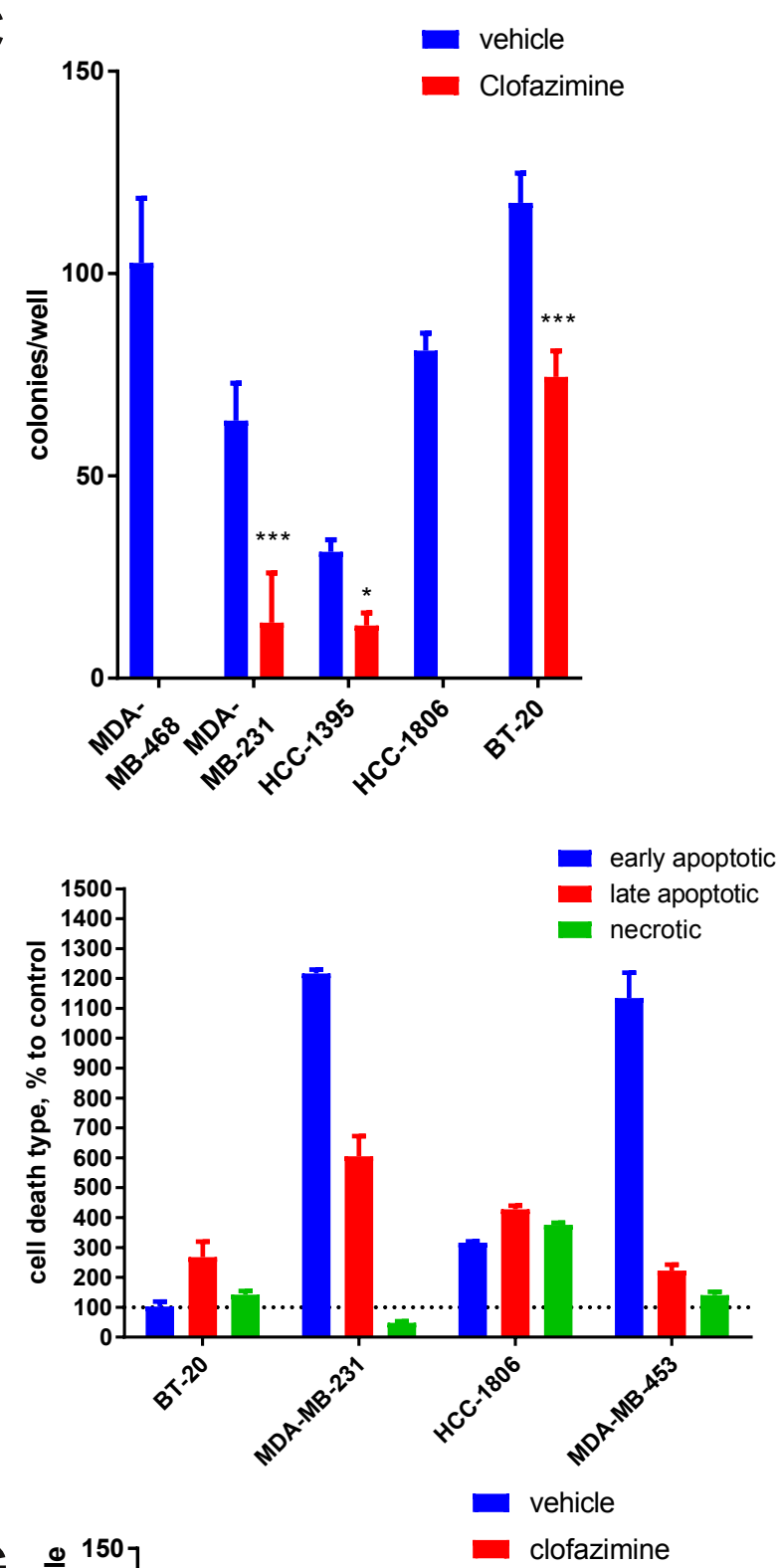

G

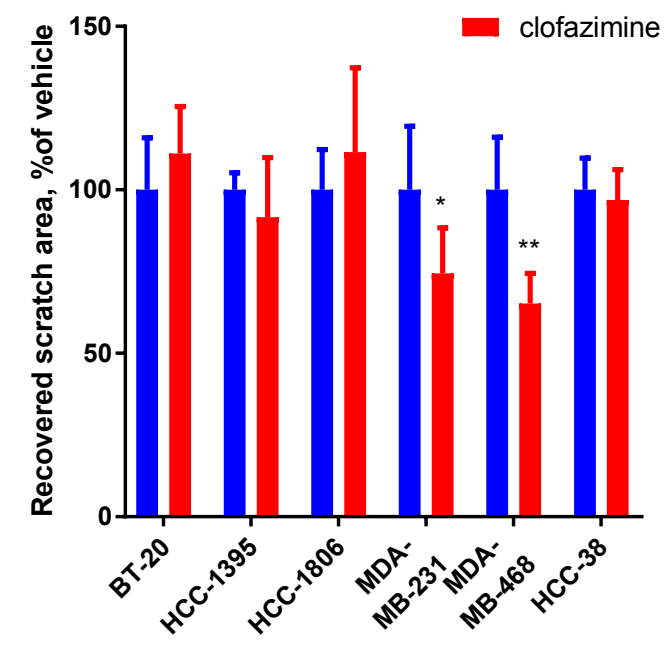

B

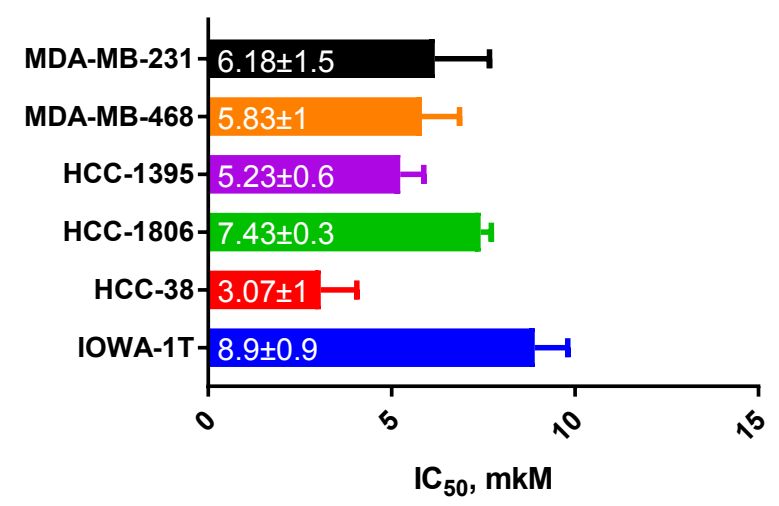

D vehicle clofazimine
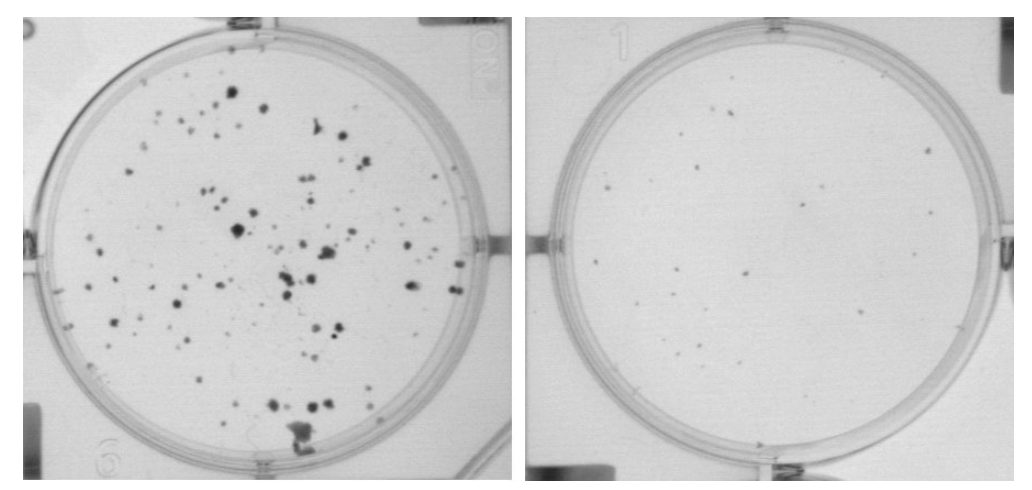

F

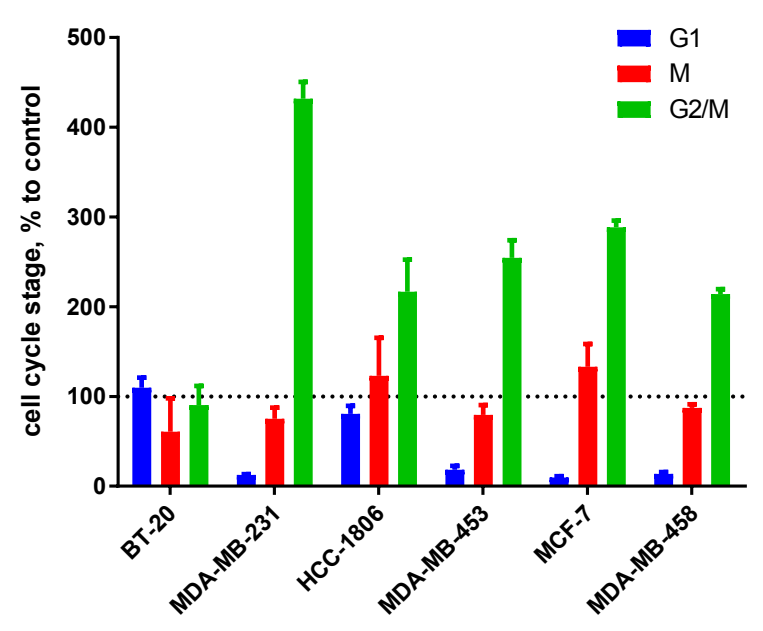

H

vehicle

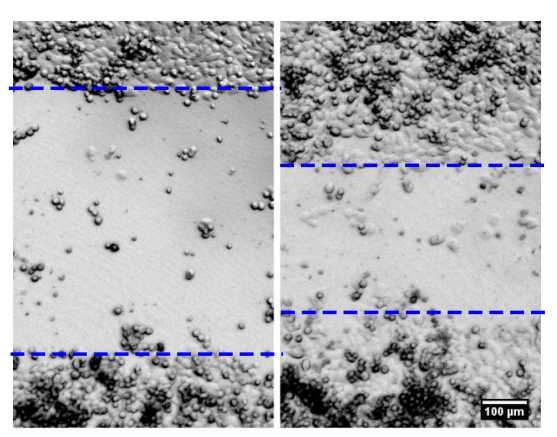

clofazimine

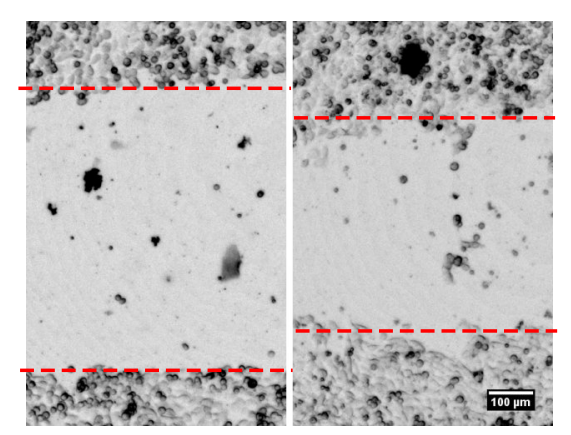


A

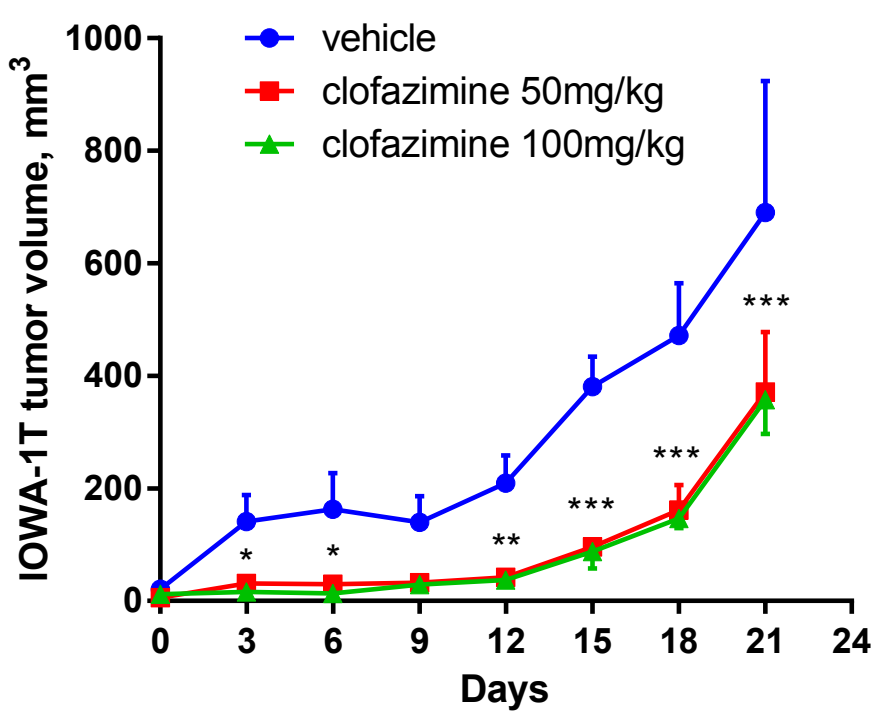

C ctrl
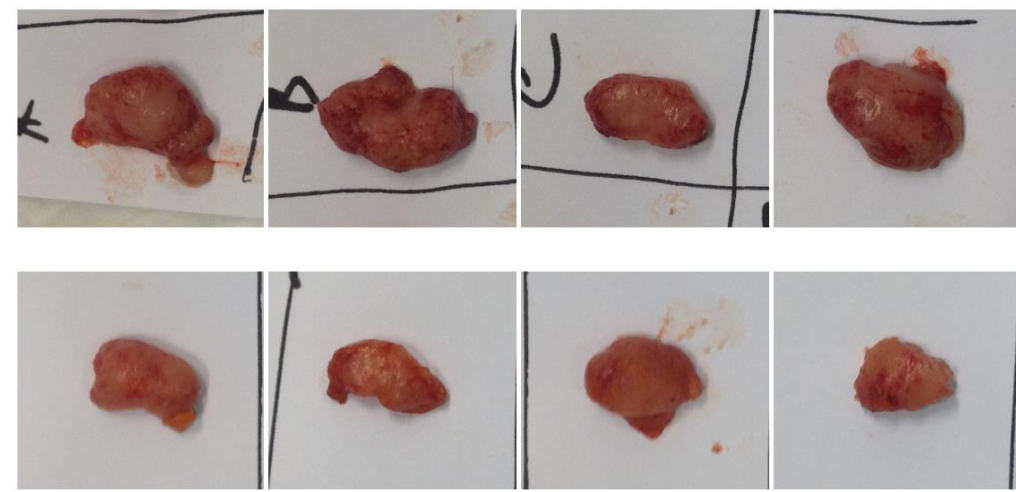

100

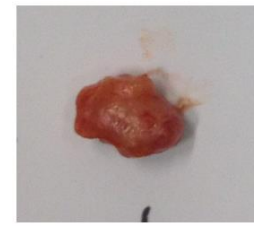

E

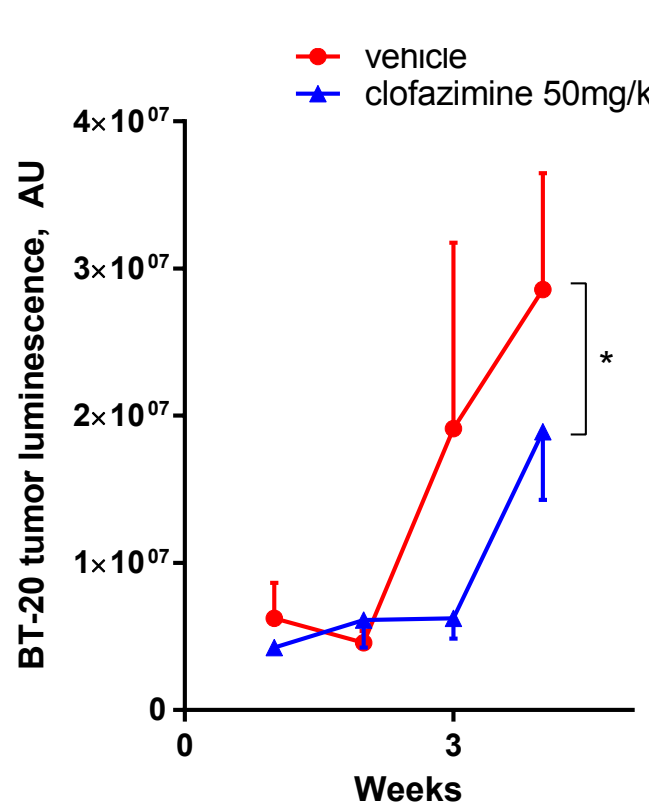

H

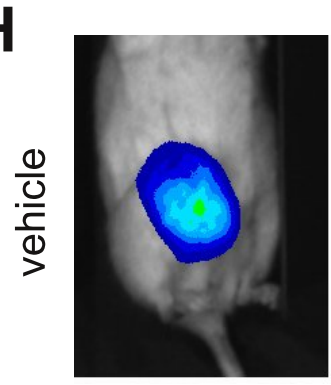

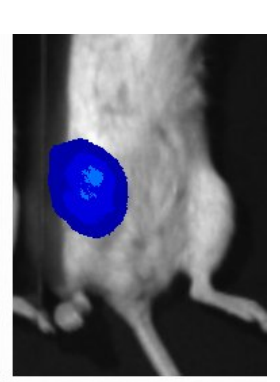
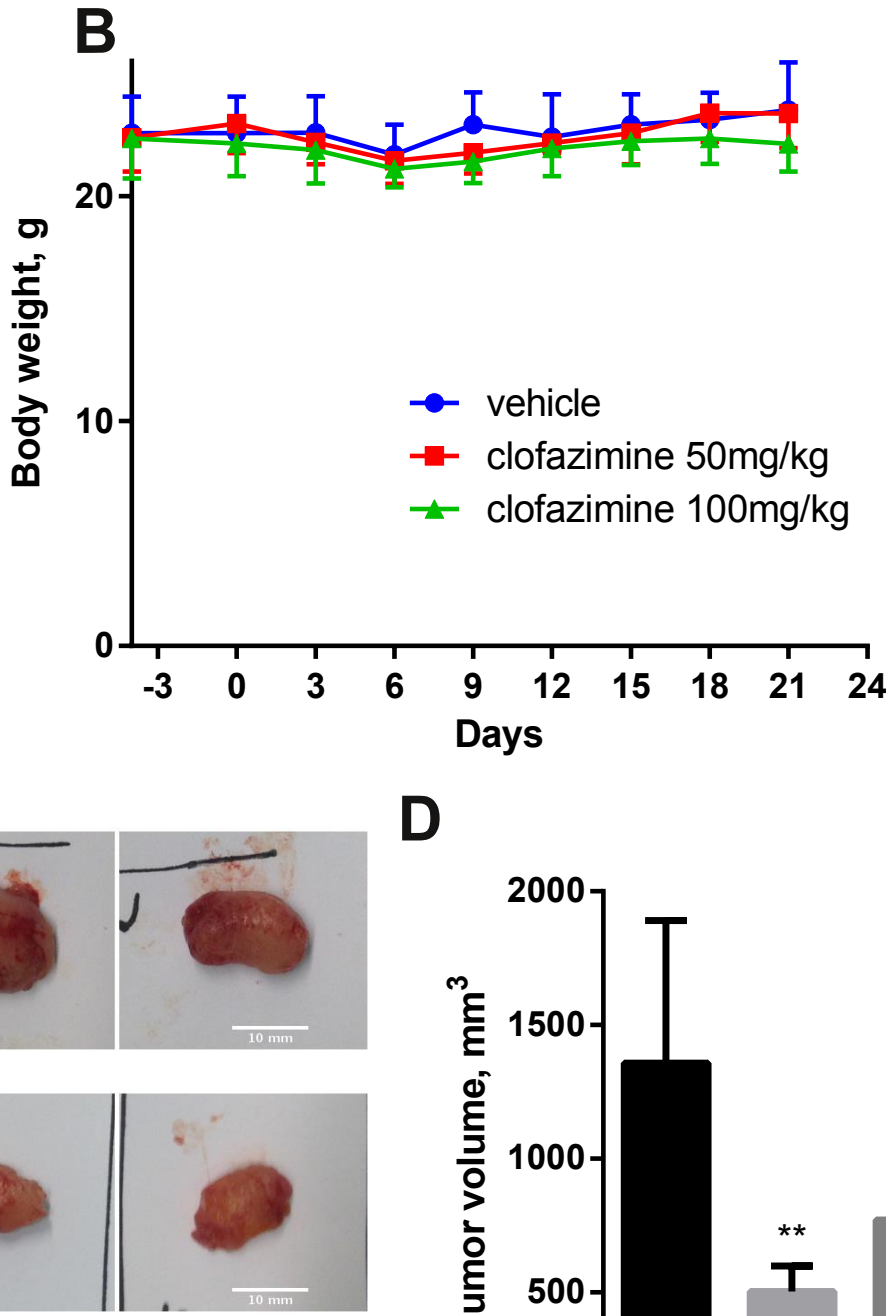

D

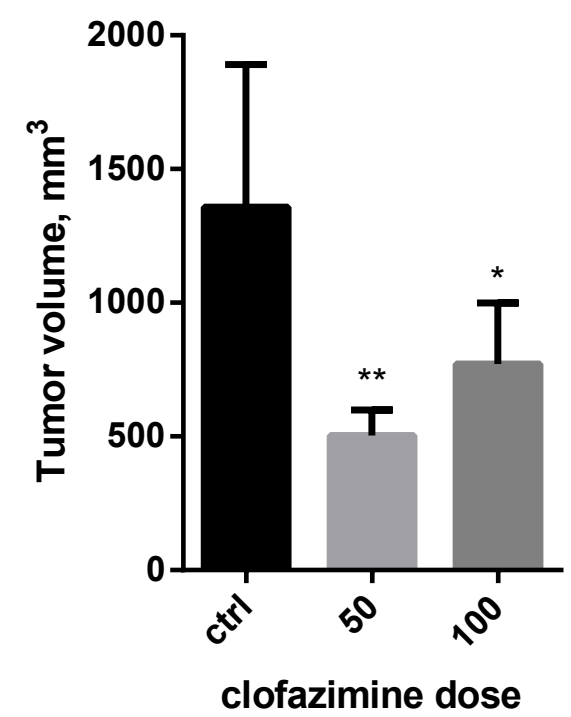

F
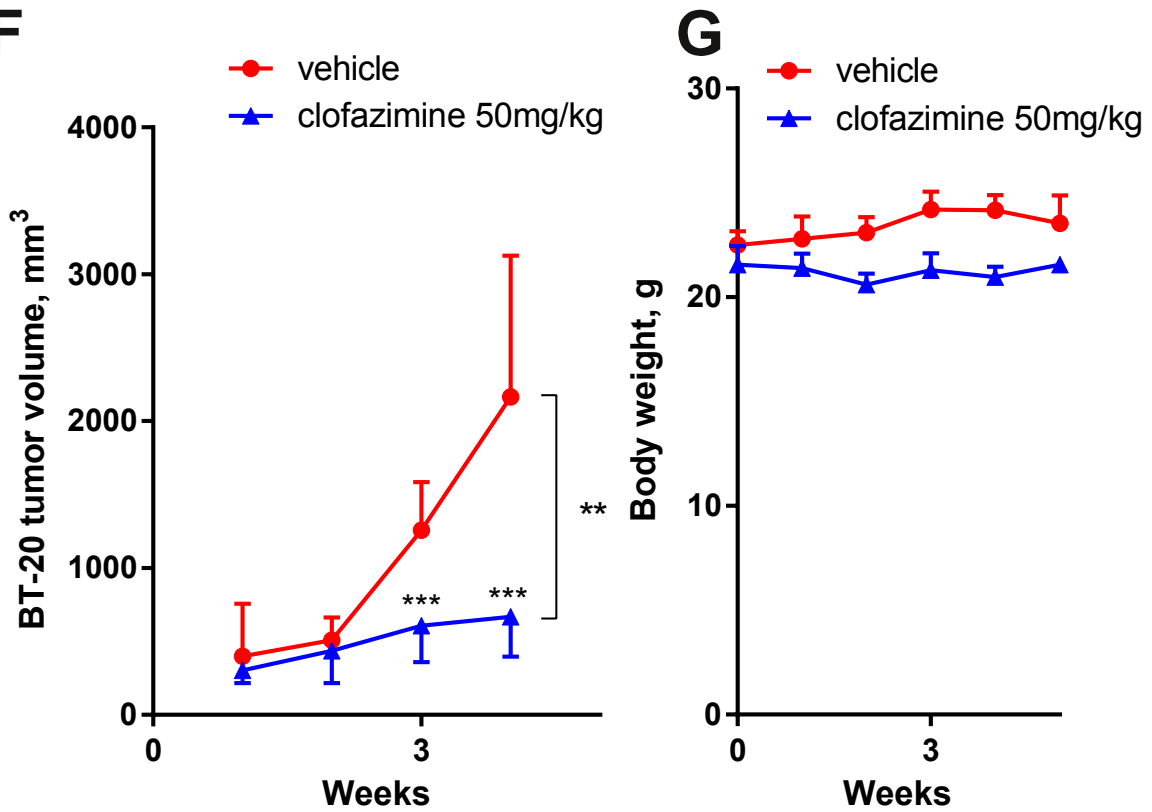
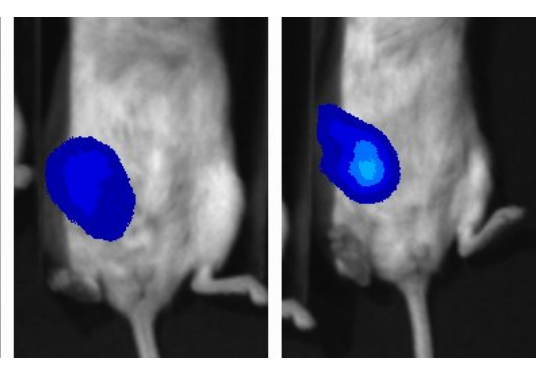

Ahmed et al, Figure 3 

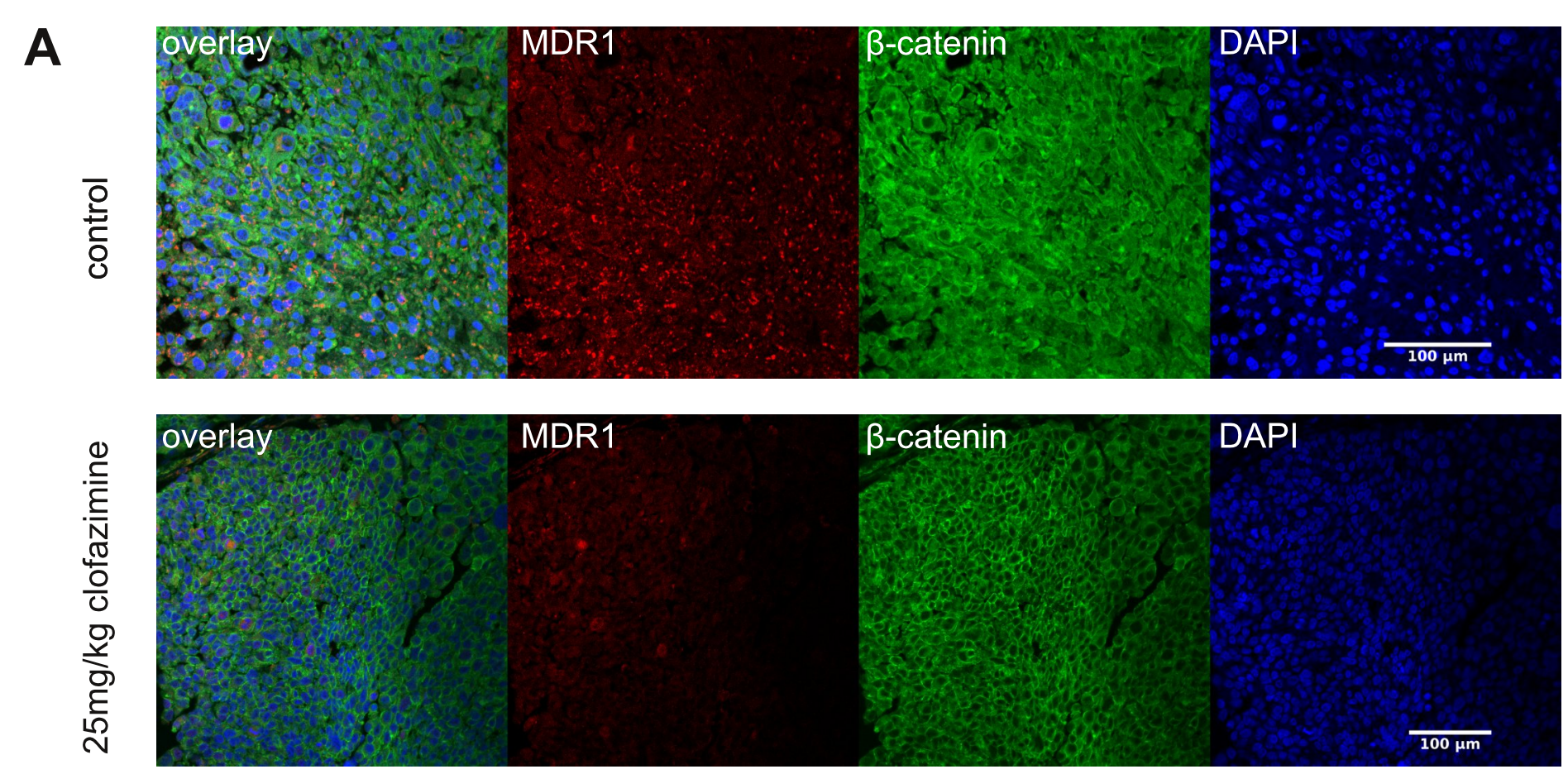

B

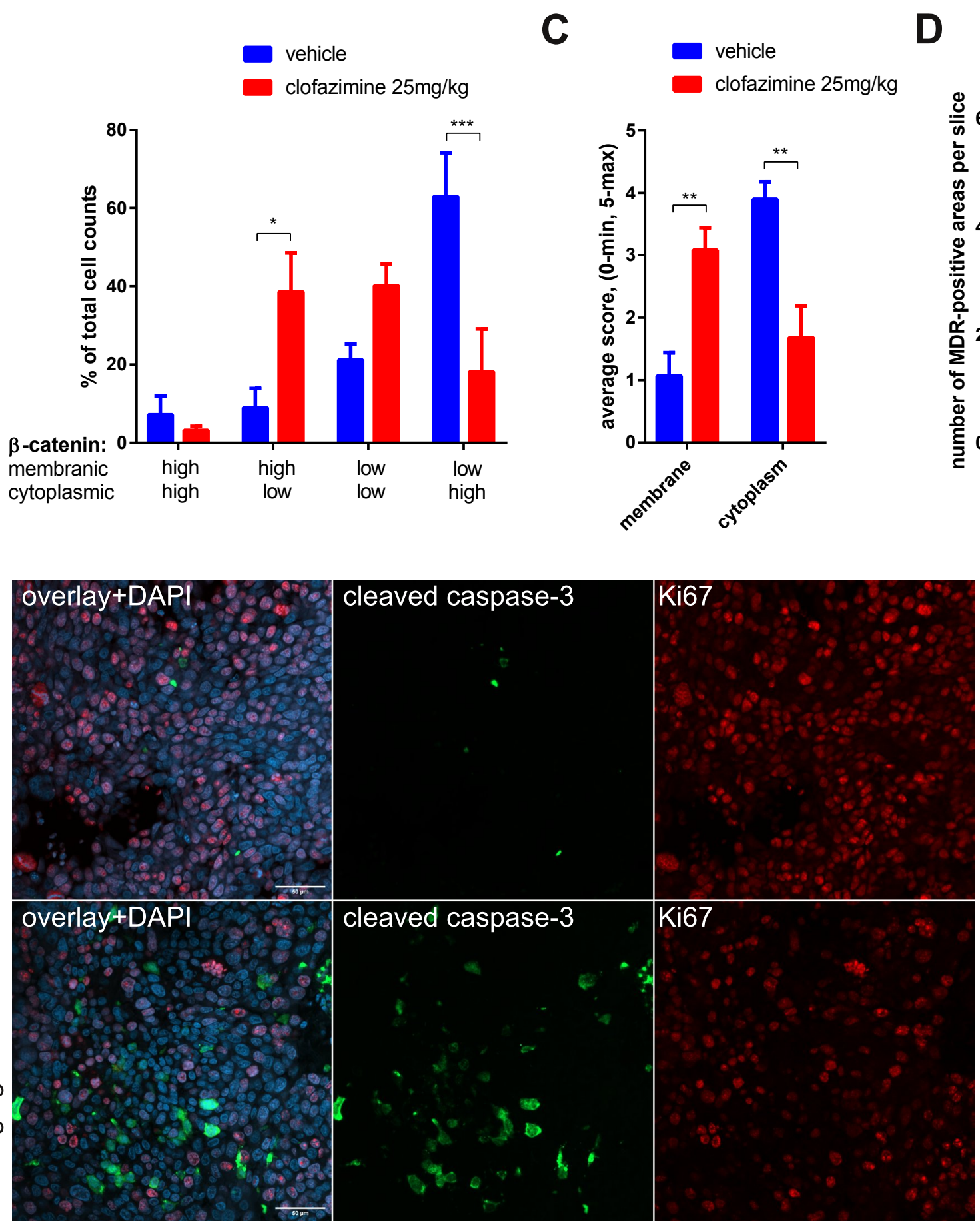

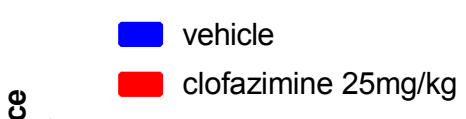

$F$
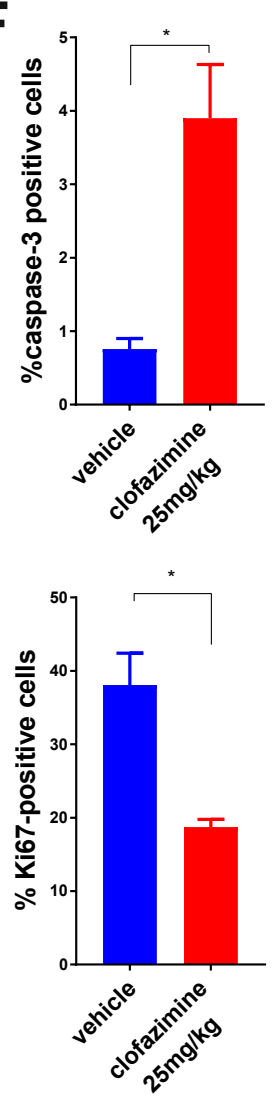


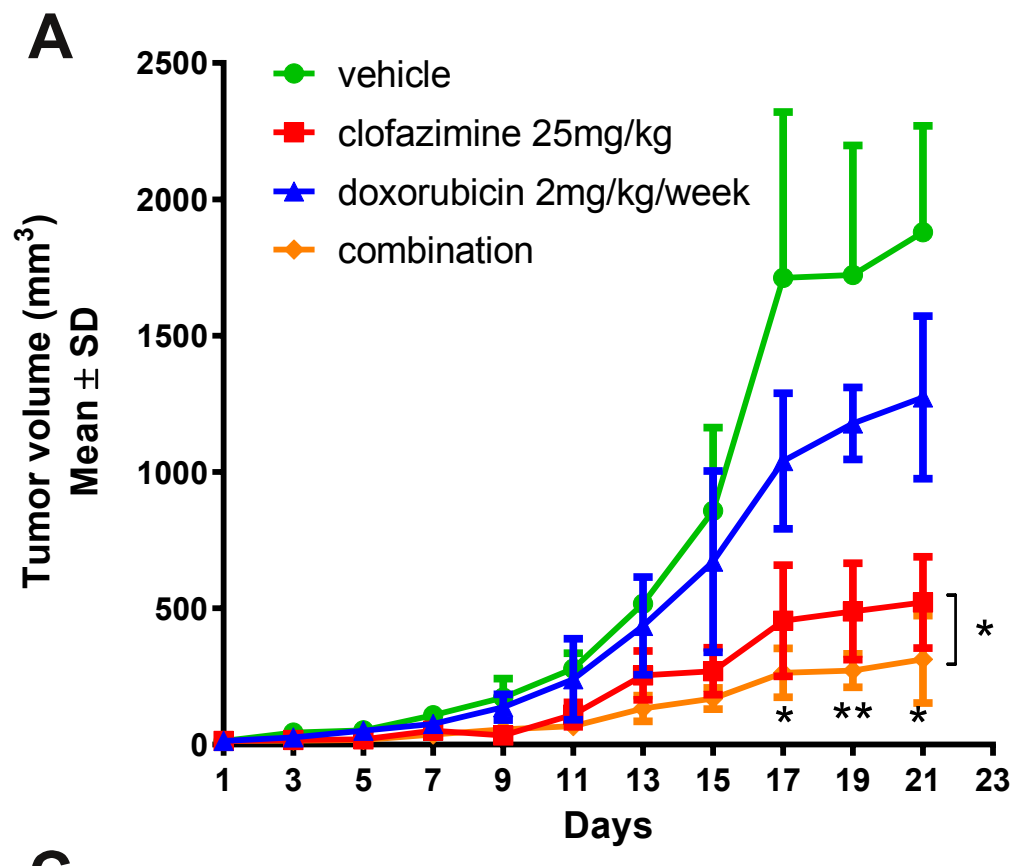

B

C

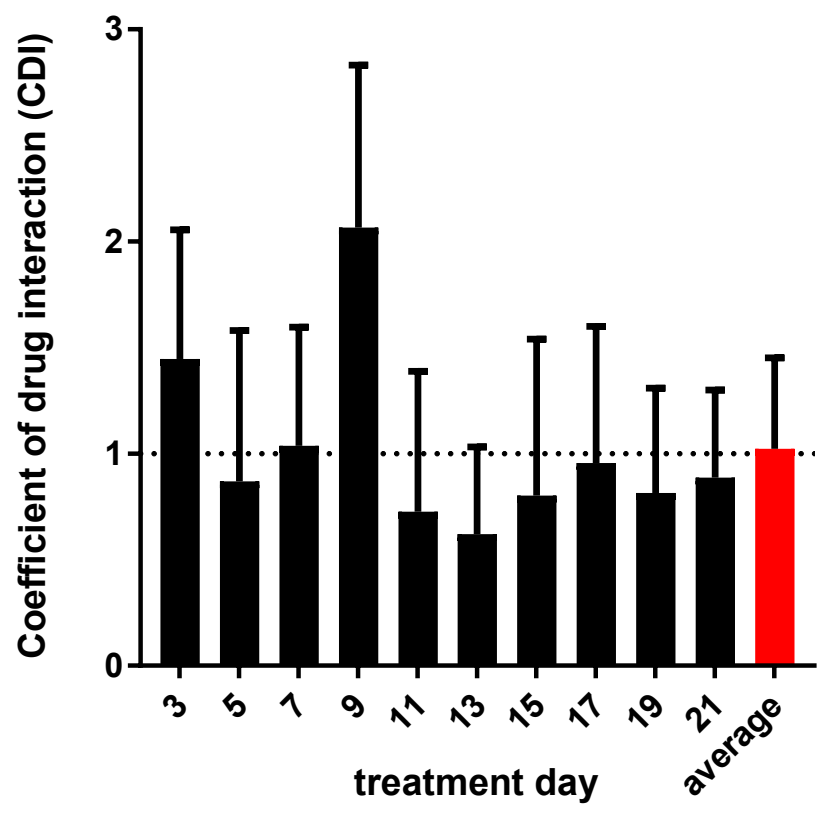

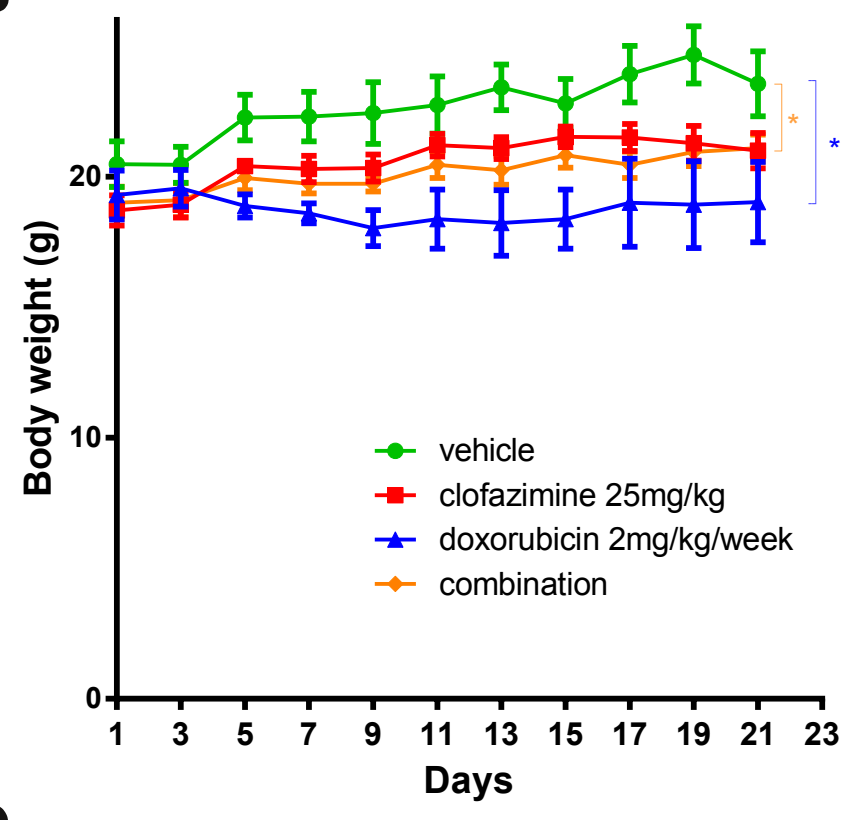

D

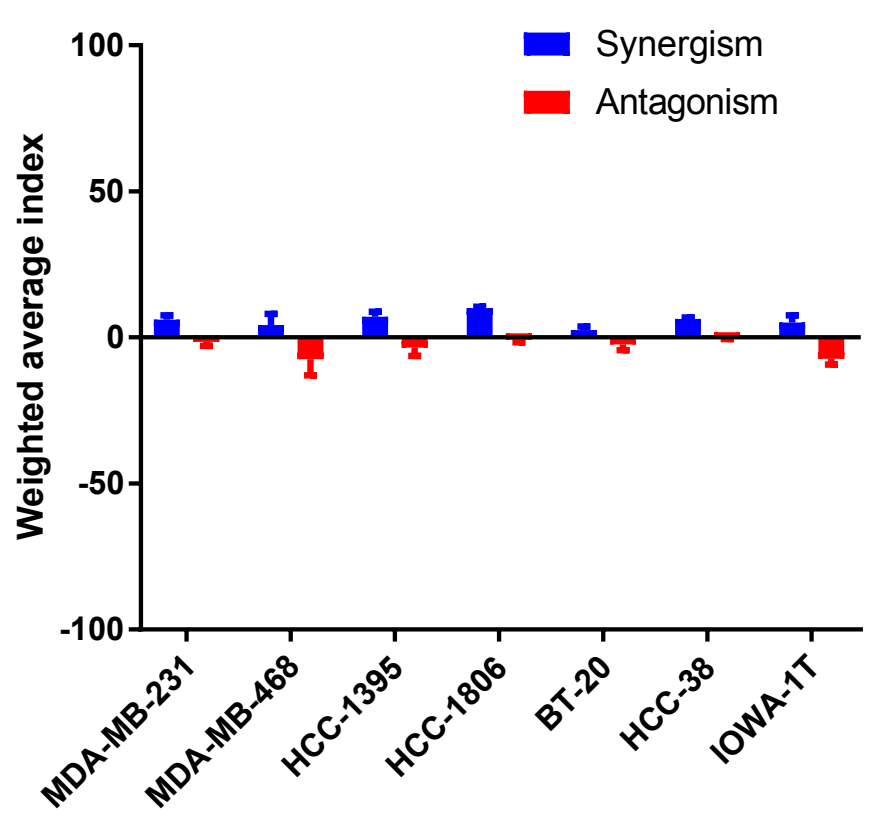


A
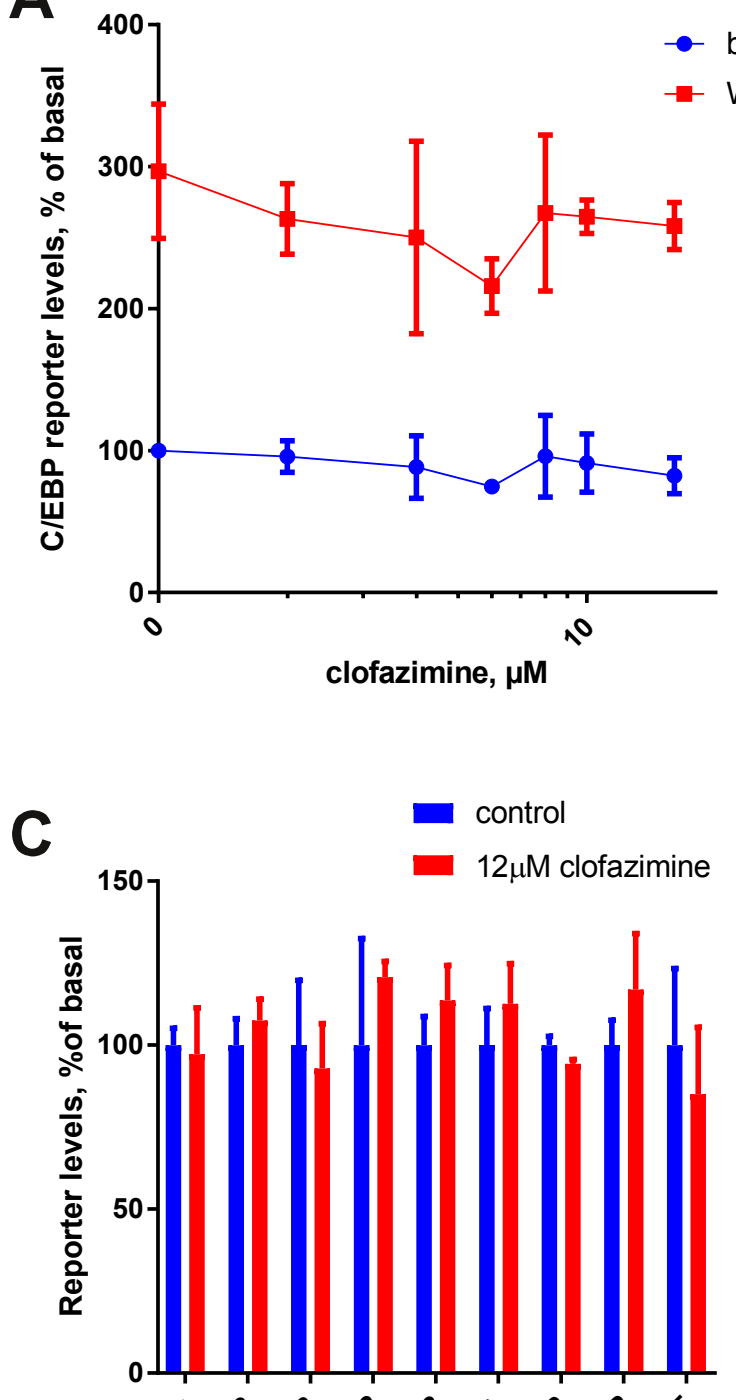

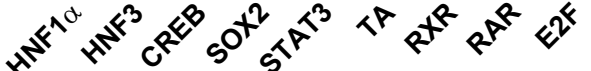

pathway reporter

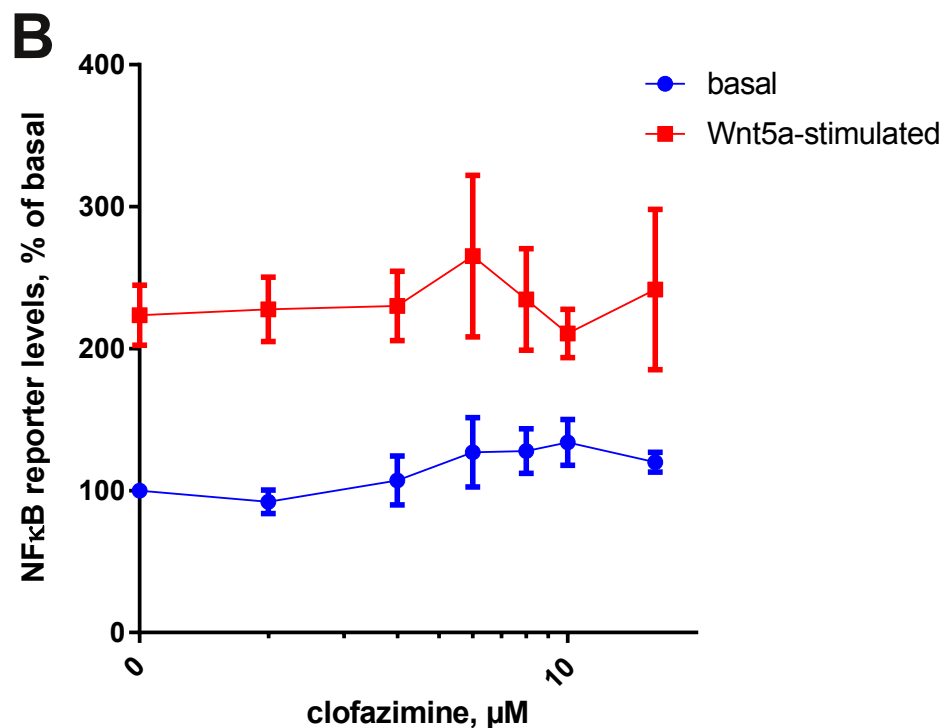

Supplementary Figure 1. Clofazimine effects were analyzed for two noncanonical Wnt readouts (C/EBP and NFkB, both basal and Wnt5a-stimulated) and for the set of nine other pathway reporters. Statistical assessment by 2-way ANOVA indicates no statisticaly significant effects of clofazimine on any of these pathways. 


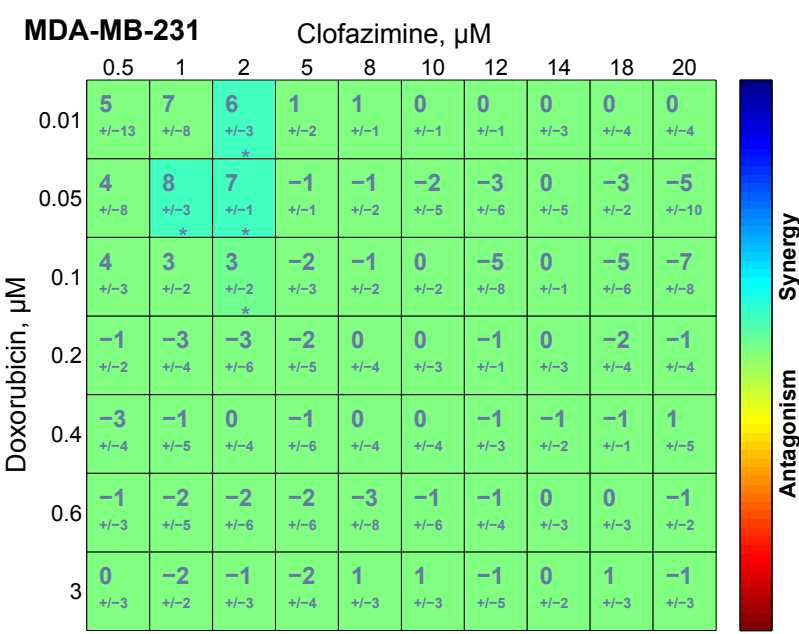

Loewe synergy and antagonism

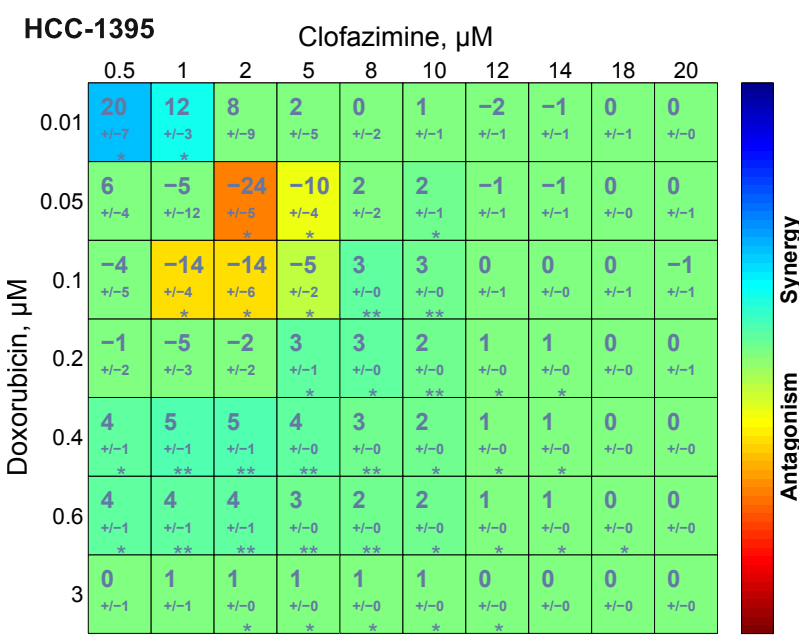

Loewe synergy and antagonism

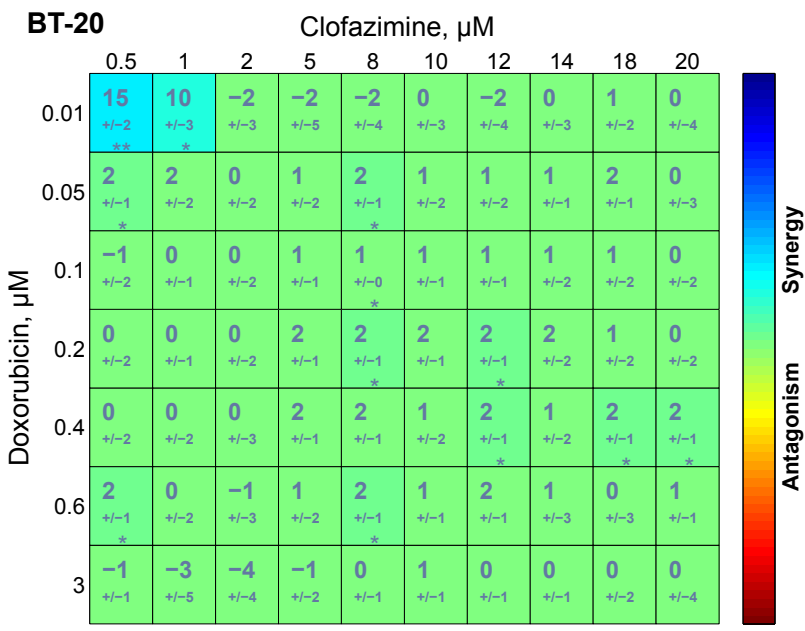

Loewe synergy and antagonism

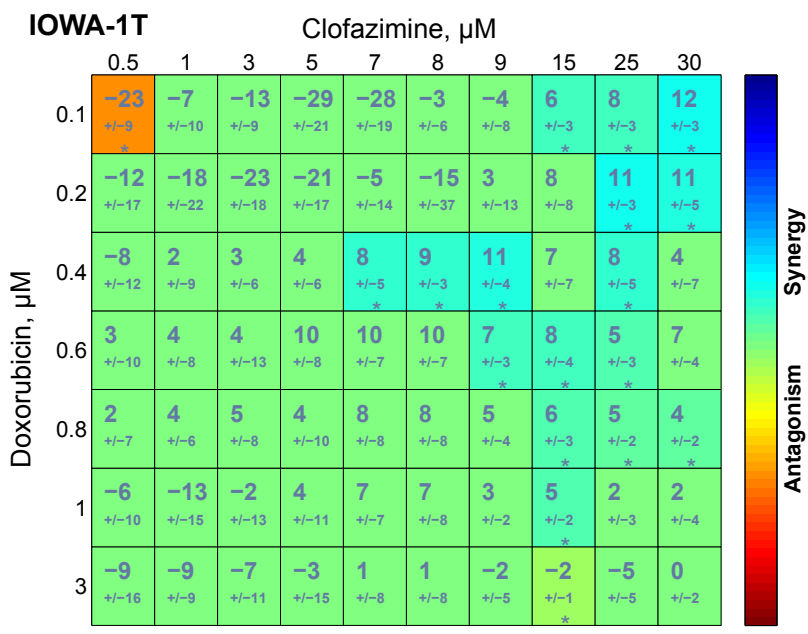

Loewe synergy and antagonism

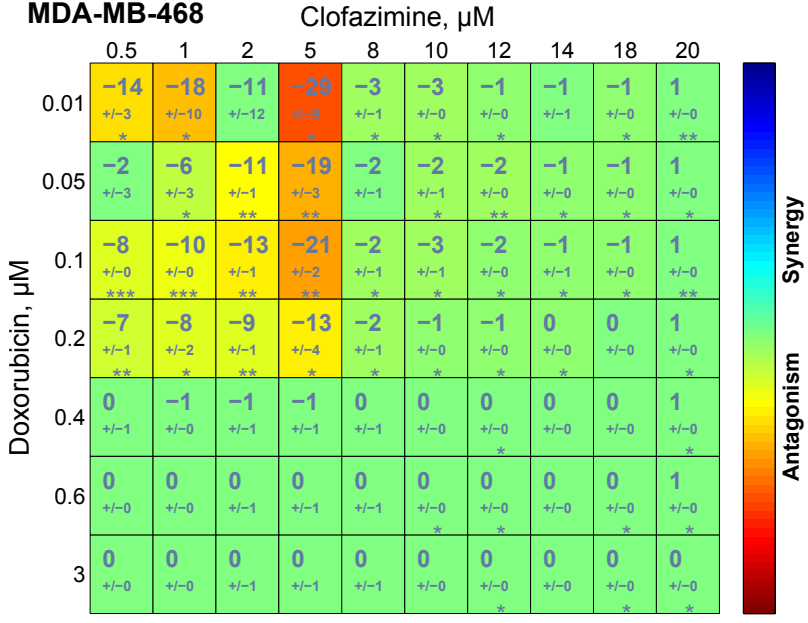

Loewe synergy and antagonism

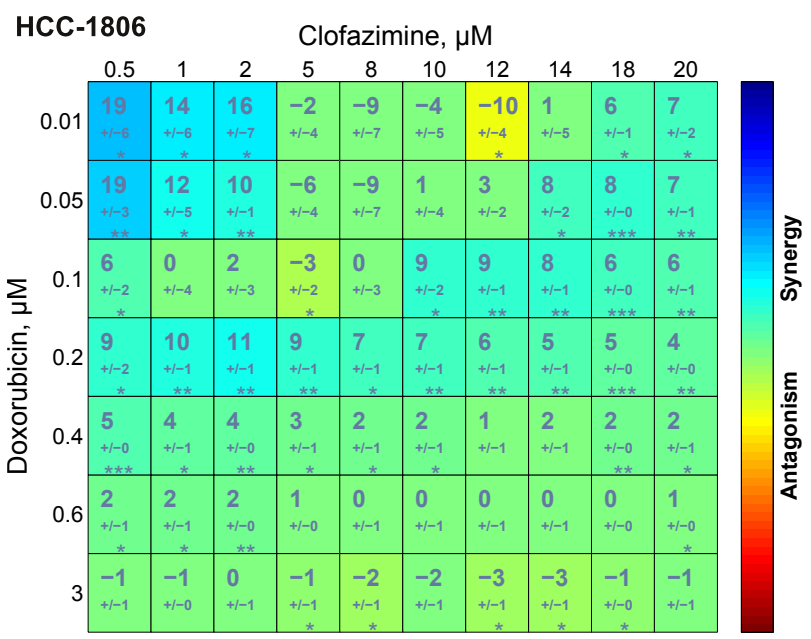

Loewe synergy and antagonism

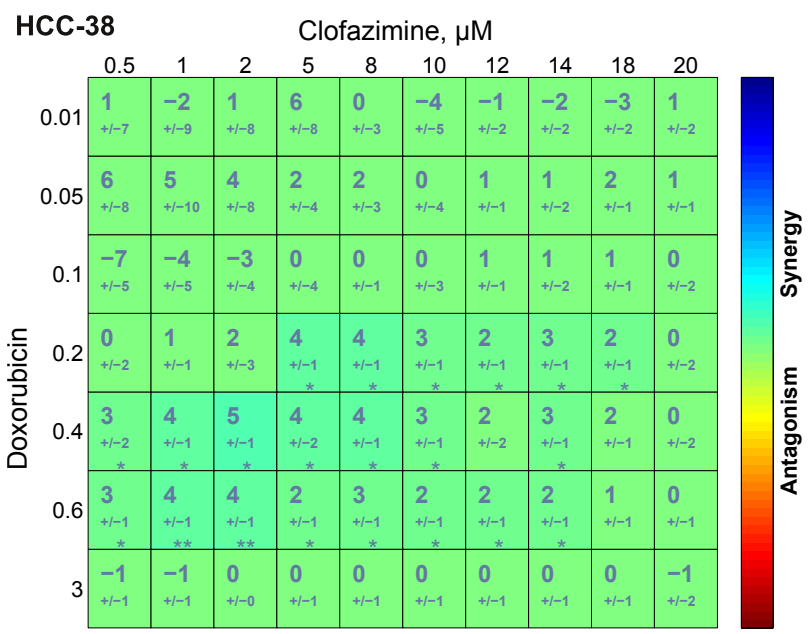

Loewe synergy and antagonism

Supplementary Figure 3. Drug interaction analysis matrices of representative experiments on corresponding TNBC cell lines obtained by Combenefit software. For most of the cell lines the magnitude of the observed effects is small and occurs in the narrow concentration range. Summary of the data is shown on Fig. 5D. 

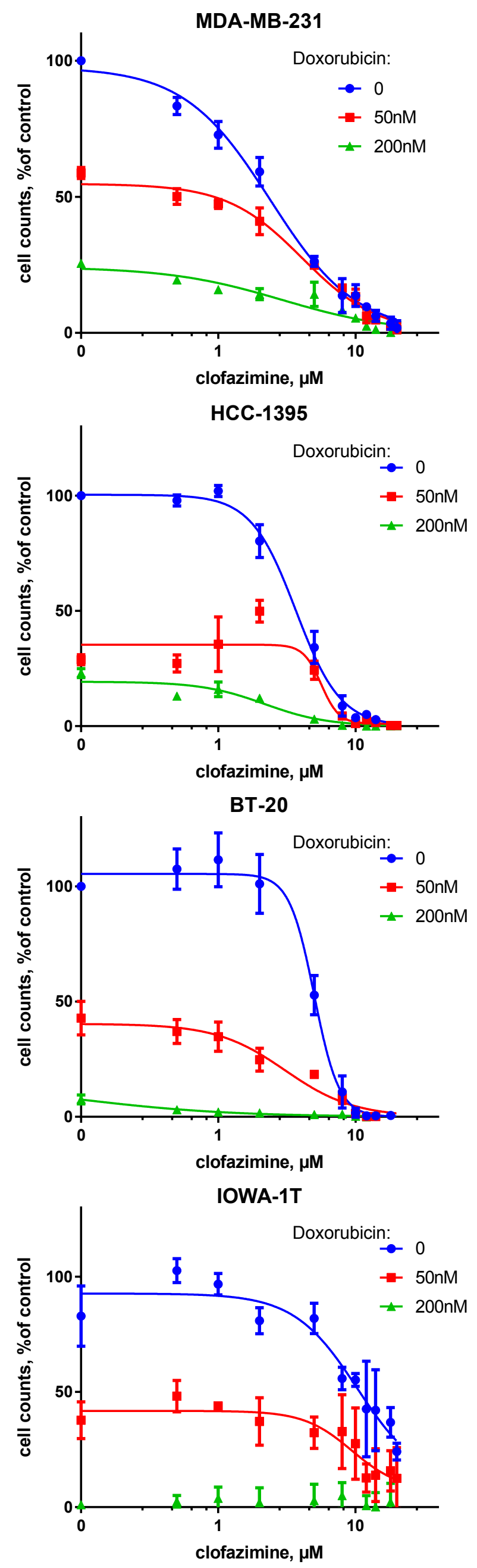

MDA-MB-468
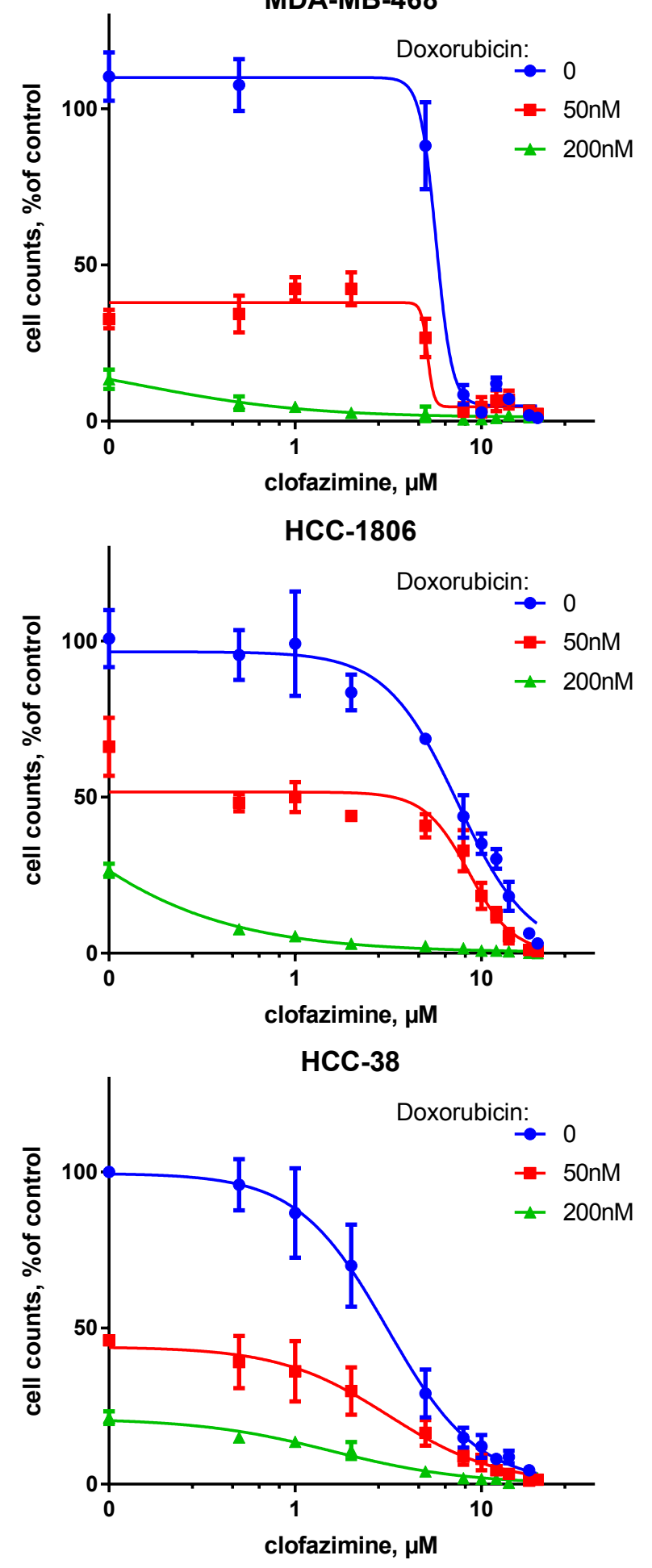

Supplementary Figure 4. Representative surivival curves of the drug combination study showing effects of doxorubicin on clofazimine dose-response curves. Summary of the data is shown on Figure 5D and combination analysis on Supplementary Fig. 2. 
Supplementary Table 1. Scoring of the adverse reactions (see Supplementary Table 2) in mice during the course of the combination study.

\begin{tabular}{|c|c|c|c|c|c|c|c|c|c|}
\hline & \multirow{2}{*}{ Groups } & \multicolumn{8}{|c|}{ Days } \\
\hline & & 0 & 3 & 6 & 9 & 12 & 15 & 18 & 21 \\
\hline \multirow{4}{*}{ Urine } & vehicle & \multicolumn{8}{|c|}{ Normal (score 0) } \\
\hline & doxorubicin & \multicolumn{8}{|c|}{ Normal (score 0) } \\
\hline & clofazimine & \multicolumn{8}{|c|}{ Normal (score 0) } \\
\hline & combination & \multicolumn{8}{|c|}{ Normal (score 0) } \\
\hline \multirow{4}{*}{ Faeces } & vehicle & \multicolumn{8}{|c|}{ Normal (score 0) } \\
\hline & doxorubicin & \multicolumn{8}{|c|}{ Normal (score 0) } \\
\hline & clofazimine & \multicolumn{8}{|c|}{ Normal (score 0) } \\
\hline & combination & \multicolumn{8}{|c|}{ Normal (score 0) } \\
\hline \multirow{4}{*}{ Skin } & vehicle & \multicolumn{8}{|c|}{ Normal (score 0) } \\
\hline & doxorubicin & \multicolumn{8}{|c|}{ Normal (score 0) } \\
\hline & clofazimine & \multicolumn{8}{|c|}{ Normal (score 0) } \\
\hline & combination & \multicolumn{8}{|c|}{ Normal (score 0) } \\
\hline \multirow{4}{*}{ Orifices } & vehicle & \multicolumn{8}{|c|}{ Normal (score 0) } \\
\hline & doxorubicin & \multicolumn{8}{|c|}{ Normal (score 0) } \\
\hline & clofazimine & \multicolumn{8}{|c|}{ Normal (score 0) } \\
\hline & combination & \multicolumn{8}{|c|}{ Normal (score 0) } \\
\hline \multirow{4}{*}{ Posture } & vehicle & \multicolumn{8}{|c|}{ Normal (score 0) } \\
\hline & doxorubicin & \multicolumn{8}{|c|}{ Normal (score 0) } \\
\hline & clofazimine & \multicolumn{8}{|c|}{ Normal (score 0) } \\
\hline & combination & \multicolumn{8}{|c|}{ Normal (score 0) } \\
\hline \multirow{4}{*}{ Conscience } & vehicle & \multicolumn{8}{|c|}{ Normal (score 0) } \\
\hline & doxorubicin & & & & Normal & & & & \\
\hline & clofazimine & & & & Normal & & & & \\
\hline & combination & & & & Normal & & & & \\
\hline & vehicle & & & & Normal & & & & \\
\hline Recniration & doxorubicin & & & & Normal & & & & \\
\hline respiration & clofazimine & & & & Normal & & & & \\
\hline & combination & & & & Normal & & & & \\
\hline
\end{tabular}

Supplementary Table 2. Scoring sheet used during the study:

Score

Monitored factor

Urine

Normal

Normal

Moist faeces

Decreased activity, slight abnormalities of posture or activity,
aggression, fear
General condition (skin, orifices, posture and conscience) of posture or activity
aggression, fear

All factors normal
Changes in color and appearance
2

3

Severe hematuria, severe incontinence, no urination for period of $>24 \mathrm{~h}$

Severe or bloody diarrhea excessive dryness

Severe loss of activity, poor grooming, skin dehydration
Near or complete paralysis, cachexia or emaciation 
${ }^{*}$ Conflicts of Interest Statement

Click here to download Conflicts of Interest Statement: Competing interests.docx

Competing interests: The authors declare that they have no competing interests. 